\title{
Effectiveness of Virtual/Augmented Reality-Based Therapeutic Interventions on Individuals With Autism Spectrum Disorder: A Comprehensive Meta-Analysis
}

\author{
Behnam Karami $i^{1,2,3}$, Roxana Koushki ${ }^{1,3}$, Fariba Arabgol ${ }^{3,4}$, Maryam Rahmani $^{3}$ and \\ Abdol-Hossein Vahabie ${ }^{5,6 *}$ \\ ${ }^{1}$ School of Cognitive Sciences, Institute for Research in Fundamental Sciences (IPM), Tehran, Iran, ${ }^{2}$ Cognitive Neuroscience \\ Laboratory, German Primate Center, Goettingen, Germany, ${ }^{3}$ School of Medicine, Shahid Beheshti University of Medical \\ Sciences, Tehran, Iran, ${ }^{4}$ Behavioral Science Research Center, Shahid Beheshti University of Medical Sciences, Tehran, Iran, \\ ${ }^{5}$ Department of Psychology, Faculty of Psychology and Education, University of Tehran, Tehran, Iran, ${ }^{6}$ Control and Intelligent \\ Processing Center of Excellence (CIPCE), Cognitive Systems Laboratory, School of Electrical and Computer Engineering, \\ College of Engineering, University of Tehran, Tehran, Iran
}

OPEN ACCESS

Edited by:

Raz Gross,

Sheba Medical Center, Israel

Reviewed by:

Yoon Phaik Ooi,

University of Basel, Switzerland

Diogo Morais,

Lusophone University of Humanities

and Technologies, Portugal

*Correspondence:

Abdol-Hossein Vahabie

h.vahabie@ut.ac.ir

Specialty section:

This article was submitted to Child and Adolescent Psychiatry,

a section of the journal

Frontiers in Psychiatry

Received: 07 February 2021 Accepted: 12 May 2021

Published: 23 June 2021

Citation:

Karami B, Koushki R, Arabgol F, Rahmani $M$ and Vahabie A-H (2021) Effectiveness of Virtual/Augmented

Reality-Based Therapeutic

Interventions on Individuals With

Autism Spectrum Disorder: A

Comprehensive Meta-Analysis.

Front. Psychiatry 12:665326.

doi: 10.3389/fpsyt.2021.665326
In recent years, the application of virtual reality (VR) for therapeutic purposes has escalated dramatically. Favorable properties of VR for engaging patients with autism, in particular, have motivated an enormous body of investigations targeting autism-related disabilities with this technology. This study aims to provide a comprehensive meta-analysis for evaluating the effectiveness of VR on the rehabilitation and training of individuals diagnosed with an autism spectrum disorder. Accordingly, we conducted a systematic search of related databases and, after screening for inclusion criteria, reviewed 33 studies for more detailed analysis. Results revealed that individuals undergoing VR training have remarkable improvements with a relatively large effect size with Hedges $g$ of 0.74 . Furthermore, the results of the analysis of different skills indicated diverse effectiveness. The strongest effect was observed for daily living skills ( $g=1.15)$. This effect was moderate for other skills: $g=0.45$ for cognitive skills, $g=0.46$ for emotion regulation and recognition skills, and $g=0.69$ for social and communication skills. Moreover, five studies that had used augmented reality also showed promising efficacy ( $g=0.92)$ that calls for more research on this tool. In conclusion, the application of VR-based settings in clinical practice is highly encouraged, although their standardization and customization need more research.

Keywords: autism spectrum disorder, virtual reality, rehabilitation, technology, augmented reality

\section{INTRODUCTION}

Autism spectrum disorder (ASD) is a complex neurodevelopmental disorder characterized by impairments in social communication and social interaction in conjunction with restricted, repetitive patterns of behavior, interests, or activities (1). Affecting 1 in 68, ASD is the most prevalent psychological childhood disorder with sustained long-term effects on the quality of life of these patients (2). 
Although at present there is no particular accepted treatment for ASD, there is a growing consensus that appropriately targeted individualized behavioral and educational intervention programs [e.g., Treatment and Education of Autistic and Related Communication Handicapped Children (TEACCH) program, Early Intensive Behavioral program, Applied Behavior Analytic (ABA) program, Denver model, etc.] have the potential to positively impact the lives of individuals and their families (3-7). The increasing number of individuals with ASD together with the substantial achievements that have been made thus far by this behavioral rehabilitation programs has ignited a line of research aimed at developing several technologies with the focus on improving these programs (8). Some examples include robotics (9-11), interactive video modeling (12-14), mobile and touchpad devices (15-17), wearable training systems on Google Glass (18), and virtual reality $(\mathrm{VR})(19,20)$. Interestingly, individuals with ASD have shown special interest and adherence to computerized programs (21) and learning through it $(22,23)$. Moreover, the burden of many hours of training by a therapist can be alleviated by using technology-based training at home.

Among these technologies, VR has become one of the most promising tools to address the psychological needs of people with ASD in various settings. Since two decades ago, VR was introduced as an effective tool in the neurocognitive rehabilitation of patients with ASD (24). This effectiveness has been approved by a decade of research afterward practicing different types of VR configurations on patients with different levels of disorder $(25,26)$. Besides, some efforts could have possibly improved the application of VR technology in recent works by proposing consideration of psychological theories in task design (27) and highlighting particular features of VR configurations and human-VR interactions (28). VR reduces the social pressure on the patient and provides a realistic environment for more effective training and possibly reduces the needed training hours. Current studies cover a great range of training interventions, including training of social adaptation and communication skills (29-31); emotional skills (32-34); daily living skills such as shopping $(35,36)$, driving (37-39), and street crossing (40, 41); and cognitive functions (42-44).

$\mathrm{VR}$ is a human-computer interface, which by using computer graphics generates a multidimensional environment with multiple sensory channels that allow individuals to explore the virtual environment (VE) through visual, auditory, tactile, and sometimes even olfactory perception, creating an interactive and immersive experience for the user $(45,46)$. VR can be implemented in head-mounted visual display (HMD) systems, head and body tracking, CAVE (Cave Automatic Virtual Environment) automatic VEs or room-like displays, and other technologies. They can be used to create a realistic sense of "presence" within a computer-generated environment (47). Augmented reality (AR), which can be considered as another type of $\mathrm{VR}$, is a real-time view of an existing world that is superimposed by some virtual data. Unlike VR technology that fully submerges people in an artificial environment avoiding the existing world, AR technology enhances the feeling by overlaying the computer-generated things over the real world (48).
VR training offers several advantages; perhaps the most important one is to provide a safe access to realistic environments that would be considered dangerous in the real world along with active participation in the virtual world. Furthermore, by providing flexibility in controlling the task complexity, reinforcement through repetition and real-time visual and auditory feedback, VR enhances enjoyment and thus improves learning quality through it. These favorable properties of VR have made it a viable tool to be used in training and rehabilitation $(49,50)$.

In the past decade, VR has served as an effective new treatment tool in different areas such as rehabilitation in post-stroke patients $(51,52)$, pain management (53), phobias, posttraumatic stress disorders, obsessive-compulsive disorders, anxiety and stress disorders (54), depression (55), attentiondeficit/hyperactivity disorder (ADHD) (56), cerebral palsy (57), and of course, ASD.

Although during recent years, several systematic reviews have been conducted to evaluate the efficacy of technology application on training and teaching different skills such as communication and social skills $(58,59)$, academic skills $(60)$, or information processing (61), only the contribution of Mesa-Gresa et al. (62) was focused on VR and autism as an evidence-based systematic review on the effectiveness of VR-based intervention in ASD. However, their study did not provide a statistical analysis of outcomes for different clinical targets; besides, the included population in their study was limited to children and adolescents.

To date of this study, there is only one meta-analysis on technology-based intervention such as computer games, interactive DVDs, shared active surfaces, and VR in patients with autism (63). Their study presented a comprehensive metaanalysis on the technology-based intervention used in ASD people; however, the type of technology used in their included studies was mostly based on computer gaming software. Since the time of that study, the number of studies applying VR technology for training patients with autism has witnessed a dramatic surge.

Hence, we have tried to conduct a comprehensive metaanalysis focused on the effectiveness of VR technology per se in the training and rehabilitation of patients with autism. To achieve this goal, we performed a systematic search for studies assessing this type of intervention on the ASD population and evaluated the effectiveness of VR training on different skills including social and communication, emotion regulation, daily living, and cognitive skills (CS). We evaluated and compared the effect sizes (ESs) of different skills' improvement to appraise the most influenced clinical targets.

\section{MATERIALS AND METHODS}

\section{Study Identification and Selection}

We systematically searched clinical and technical databases including PubMed, ERIC, Web of Science, PsycINFO, and IEEE following a comprehensive search strategy with the main search terms of virtual reality, augmented reality, artificial reality, computer-simulated reality, virtual environment, virtual world, computer-simulated environment, mediated reality, and mixed reality for intervention and the search terms of autism spectrum 


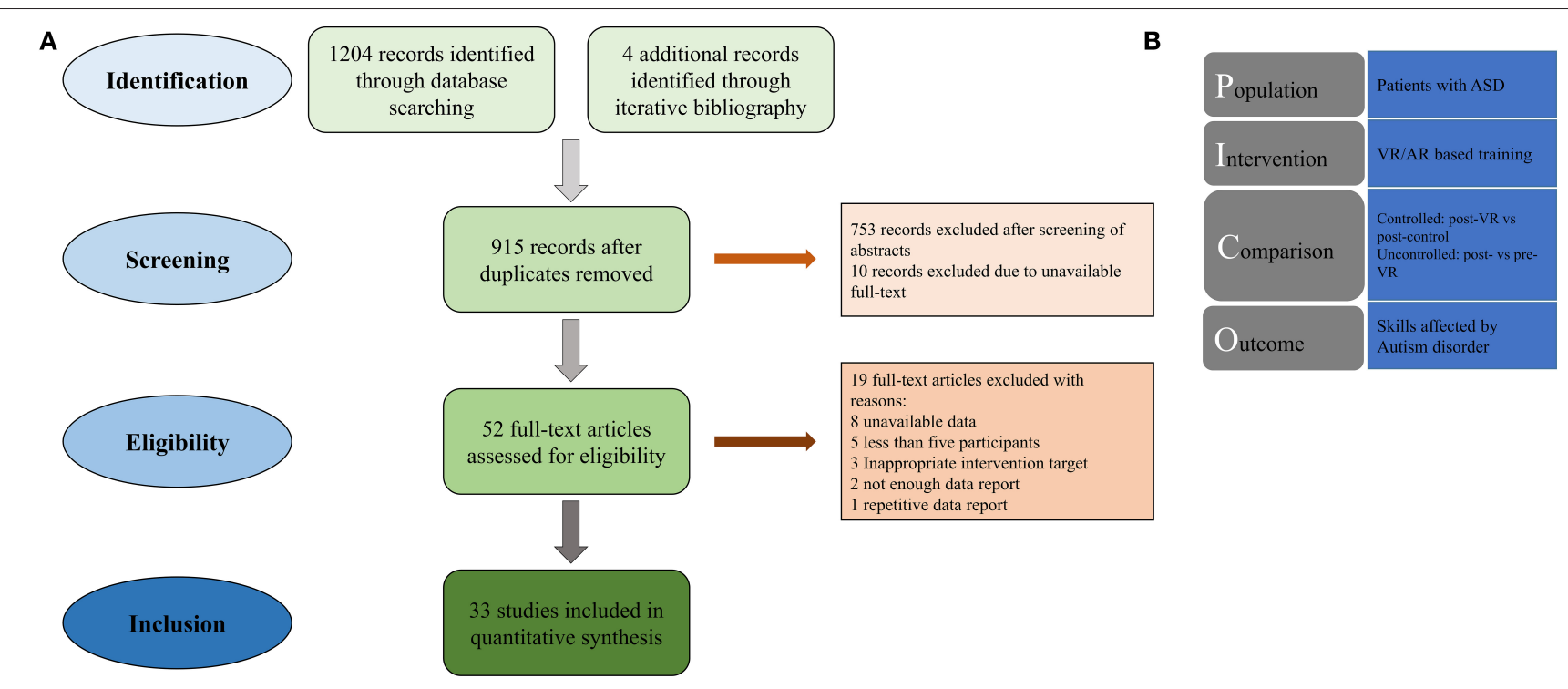

FIGURE 1 | (A) Flow diagram of study selection and identification process. (B) Schematic presentation of PICO for this study.

disorder, pervasive children developmental disorder, and Asperger for disorder, considering adjusted queries for each database. The detailed search strategy and search queries for each database can be seen in Supplementary Material. The initial search yielded a total of 1,204 articles. There was no limit on the publication date, and the search is updated until October 19, 2019.

After removing duplicate records, 915 articles remained for the preliminarily screening of titles and abstracts. Those studies presenting original work and discussed virtual or artificial realities for rehabilitation and training of the ASD population that were published in a peer-reviewed journal or peer-edited conference proceeding books were selected. Case reports, review articles, records that contained only an abstract, and records in non-English languages have been discarded. The articles left were 52 randomized clinical trials, cohort studies, and case series. The full texts of these articles were retrieved for more detailed consideration.

A full-text detailed review was done using the PICO (patient, intervention, comparison, and outcome) process (Figure 1B). The criteria for including studies in our meta-analysis were (1) participants of any age were diagnosed with ASD with a formal diagnostic tool; (2) intervention was conducted on an interactive VR-based setting; (3) the designed intervention aimed at improving skills related to the core symptoms or deficits of ASD; and (4) the same measured data were available on a control group as for the intervention group that measurements performed on the intervention group before undertaking the goal intervention or on a control group that did not receive the goal intervention; and (5) intervention outcomes were assessed by a quantitative measure that was similar for the intervention and control conditions. The studies that did not comply with these criteria were excluded. Along with those, nine records that contained incomplete and/or inaccurate outcome reports in its text or figures were contacted for further information
$(34,35,64-69)$. One of them responded (41) and thus included in the study. Besides that, five other articles were excluded for a very small sample size $(<3)(34,70-73)$ and three others for an unfavorable design of the experiment (e.g., single case reports) $(32,74,75)$. In the end, 33 studies were proven to be eligible for entering the meta-analysis. The flow diagram of the study selection process is presented in Figure 1A.

All the studies were coded for the following items: definite diagnosis of disease, diagnostic tool, mean and standard deviation of age of participants, number of participants assigned and completed the course of intervention in the target and control groups, contributing factors and modalities that experimenters controlled for inclusion of study population, concomitant comorbidities with ASD in participants, type of intervention technology (VR or AR), technical details of intervention, experiment properties and intensity of intervention, design of experiment (uncontrolled or casecontrol), purpose of experiment, and outcome measures and their descriptions.

\section{Coding and Defining Variables}

ASD is a very heterogeneous disorder. Different patients may vary hugely in levels of deficit in different aspects of cognitive functionalities. Thus, most of the studies had attempted measuring multiple outcomes for assessment of therapeutic effectiveness. Dealing with this variability in study outcomes, we categorized them into four major categories: social and communication skills (SCS; e.g., social adaptation and interaction, communication, social reciprocity, social responsiveness, negotiation skills, theory of mind), emotion recognition and regulation skills (ERS; e.g., emotion expression, affect recognition, stress, and anxiety management), daily living skills (DLS; e.g., driving, shopping, street crossing, and job interview skills), and CS (e.g., attention and concentration, 
reasoning and problem solving, executive function, language, and metacognition). By this means, we were able not only to determine the general effectiveness of VR training but also to distinguish different aspects of ASD-related disabilities in terms of benefit they receive from intervention.

There was a considerable number of trials in which outcomes were assessed by a measure that was mostly intuitive and specifically designed for that experiment (e.g., number of greeting with a friend in VE or subject performance in a face detection task, driving, cross walking, shopping task, or construction play task) rather than measures that are widely used in the field [e.g., Social Responsiveness Scale (SRS) score of social awareness, PEP-3 score affective expressions, Adaptive Behavior Assessment System score of leisure, etc.]. We classified these two types of measures as non-formal and formal, respectively, and considered it as a possible moderator of measured training effectiveness for each trial. Another presumed moderator was the type of technology used for intervention, namely, VR or AR, which are characteristically different in terms of design and application. To explore the effectiveness of the intervention at any age, we assumed four age categories of $4-8,8-12,12-16$, and older than 16 years. Each trial fell into one of these categories based on their participants' mean age. In a considerable number of trials, patients had some concomitant comorbidity along with their main disorder, ASD. To see how much this comorbidity affected the results of the intervention, we considered the presence or absence of comorbidity as another moderator and compared the results of interventions when having or not having concomitant comorbidity. These four categorical moderators were defined for further subgroup meta-analysis. The trials in which full information regarding any of these moderators was not available were excluded from analysis for that moderator.

Subgroup meta-regression was also applied to three continuous moderator variables: number of intervention sessions, sex, and publication date. These variables were defined as the number of separate sessions or visits in which intervention was applied, male ratio (number male subjects divided by the total number of subjects), and the year of publication of the study, respectively.

\section{Statistical Procedure}

Similar to the majority of studies in the literature of training effectiveness, the pool of studies in our meta-analysis included a mixture of two major types of experiment designs, namely, controlled and uncontrolled designs. In controlled or independent-group design, one group received the training, and the other group served as a control. The difference between the groups on the outcome measure was used as an estimate of the treatment effect. On the other hand, in the uncontrolled or single-group pretest posttest design, each individual was measured before and after treatment, and the difference between the individuals' scores before and after it was used as an estimate of the treatment effect.

As the characteristic distinction between these two types of designs can lead to a significant difference in estimated ES and its precision, we opted for design-specific estimations proposed by Morris and DeShon (76) for each study. For uncontrolled studies, the repeated measure ES was calculated as the mean of change from pretest to post-test scores divided by its standard deviation, which is equivalent to the $t$ statistic of paired $t$-test between two pre-test and post-test data. Then, the Standardized Mean Difference (SMD) for these trials was calculated as follows:

$$
\text { SMD }(\text { uncontrolled })=\frac{t}{\sqrt{n}}
$$

where $t$ represents $t$ statistic, and $n$ represents the number of participants. We used the $t$ statistic values provided in the contents of articles whenever possible or calculated them from the exact pretest and posttest scores.

For controlled studies, the SMD at the posttest was calculated as follows:

$$
\text { SMD }(\text { controlled })=\frac{\mu c-\mu i}{S D p o o l}
$$

where $\mu c$ represents mean of the control group, $\mu i$ represents mean of the intervention group, and SDpool is calculated as follows:

$$
\text { SDpool }=\sqrt{\frac{(N i-1) * S D i^{2}+(N c-1) * S D c^{2}}{N i+N c-2}}
$$

where $N i$ is the size of the intervention group, $N c$ is the size of the control group, $S D i$ is the standard deviation of the intervention group, and $S D c$ is the standard deviation of the control group at posttest.

For two controlled studies in which pretest data were available for both intervention and control groups, SMD was calculated as follows:

$$
\begin{aligned}
& \text { SMD (prepost controlled }) \\
& \quad=\frac{\left(\mu \text { post } t_{i}-\mu \text { prei }\right)-(\mu \text { postc }-\mu \text { prec })}{\text { SDpre }}
\end{aligned}
$$

where $\mu$ post $t_{i}$ represents the mean of intervention group scores at posttest; $\mu$ prei, the mean of intervention group scores at pretest; upostc, the mean of control group scores at posttest; $\mu$ prec, the mean of control group scores at pretest; and SDpre is again calculated with the following equation:

$$
\text { SDpre }=\sqrt{\frac{(N i-1) * S D p r e i^{2}+(N c-1) * \text { SDprec }^{2}}{N i+N c-2}}
$$

where SDprei and SDprec represent the standard deviation of the intervention and control groups' scores at the pretest, respectively (77). All of the aforementioned calculations of SMDs were done in a way that ensures the highest precision in the estimation of each experiment's ES by the available information.

The final ES indicator, Hedges $g$, then defined as the product of the output SMD and small sample correction factor $C=$ $\frac{3}{4^{*} d f+1}$ where $d f$ is degrees of freedom. ESs were calculated and reported so that a positive sign represents an improvement in the target skill. 
After the computation of ESs for each of the trials, we found that most of them reported more than one estimated value, which is called dependent nested ESs in the literature of meta-analysis. Assuming independence between estimated values for multiple outcomes in each study is usually trivial and thus obtaining a study-level ES by averaging the values within studies might lead to some useful information loss. Handling the dependency among ES estimates, three main methods have been proposed to date: multivariate meta-analysis, three-level meta-analysis, and robust variance estimation (RVE) (78). Multivariate metaanalysis is applied when one or multiple outcomes measured in each study are from a set of known and fixed outcomes across studies. The measured outcomes in our meta-analysis were highly variable from study to study, so we could not apply multivariate analysis. Because of the small sample size of the controlled trials, some of the estimated results of the three-level analysis were underpowered and unreliable, which would question drawn conclusions based on them. So, we opted for the third introduced method, RVE. It was shown that this method accommodates well the dependence arising from multiple sources simultaneously, including multiple measures and multiple treatment groups (78) and thus can be a felicitous choice for our study. Further details on the application of the RVE method on our data are described in Results.

According to the guidelines of Cohen (79), an absolute ES of $0.2-0.3$ is regarded as a small effect, $\sim 0.5$ as a medium effect, and from 0.8 on as a large effect.

Heterogeneity was assessed by Cochran $Q, I^{2}$, and $\tau^{2}$ statistics. $I^{2}$ describes the percentage of variation in studies. The smaller the $I^{2}$, the lower the level of heterogeneity among estimated values. $\tau^{2}$ statistic is also a measure of between-study variance of ESs. When $Q$ statistic is very small, the estimated $I^{2}$ is not accurate in capturing the real heterogeneity (80). In these cases, $\tau^{2}$ is more informative specifically when comparing among subgroups with low heterogeneity.

Publication bias was investigated by visual inspection of funnel plots looking for any clue of asymmetry plus Egger intercept test (81) to validate the conclusions.

All the analyses in the main text were done using customized scripts in MathWorks' MATLAB. Three-level meta-analysis was performed in $\mathrm{R}$ using an available $\mathrm{R}$ package (82).

\section{RESULTS}

\section{Description of Studies}

Thirty-three studies complied with the inclusion and exclusion criteria (see Methods) and entered into the meta-analysis.

The interventions were applied by a controlled experiment design in seven studies and by an uncontrolled design in 24 studies. There were two studies that recruited both types of controlled and uncontrolled designs (mixed-design) (83, 84). As these mixed-design studies included different participants in each design group, we treated them as separate uncorrelated trials. Doing so, we based our analysis on 35 independent trials obtained from 33 studies (The term trial refers to an independent design group consistently thereafter in this article). All in all, 540 ASD participants were included in this study, of which 360 belong to uncontrolled and 180 to controlled trials. There were also 156 ASD patients in the control arm of controlled trials who received neither VR-based nor conventional intervention.

In four controlled trials, the same outcomes were measured before (baseline) and after training in both the control and intervention arms. In the remaining five controlled trials, these measurements were done only after training, and there were no baseline data provided in any control or intervention group. Of 26 uncontrolled trials, three of them applied $\mathrm{ABC}$ measurement strategy in a way that outcomes were measured in three temporal phases: after the first session (pre), after the last session (post), and a while after completion of intervention (follow-up) $(29,44,85)$. In the other trials, the measurements were performed before (pre) and after (post) interventions. In two trials (one from control and the other one from uncontrolled trials), measurement once was done after a nonVR conventional training, and it was repeated after VRbased target training $(43,86)$. The data in the first condition were labeled pre-intervention, and in the latter labeled postintervention. A prior exposure to any type of training was neither recognized nor mentioned in the other studies. The identified pre-intervention and post-intervention data for each trial were used in computing ES statistics (see Methods for more detail).

The diagnostic tools used to integrate patients into the study were different across trials. For instance, in two trials, diagnosis was confirmed by the Diagnostic and Statistical Manual of Mental Disorders, Fourth Edition (DSM-IV) $(83,85)$, in four other trials by Autism Diagnostic Observation Schedule (ADOS) (39, 42, 87, 88), in one of them by Social Communication Questionnaire (SCQ) (89), in the other two trials by SRS-II $(90,91)$, in the other 6 by $D S M-V(41,92-94)$, and in another one by Childhood Autism Rating Scale (CARS) (40). The diagnostic tool in the remaining 20 trials was not mentioned. Regarding stage/level of disease, three trials included patients with high-functioning ASD (HFASD) $(90,94,95)$, one trial had patients with low-functioning ASD (LFASD) (92), two trials included Asperger or pervasive developmental disorder-not otherwise specified patients (31, 87), two other trials had patients with either HFASD or Asperger $(96,97)$, and one trial had patients with Asperger who received the intervention (98). The level of disease in the other 26 trials was not specified. Several trials considered controlling some contributing factors in the population of their study that could potentially impact the outcome of the intervention. For example, eight trials controlled the participants for IQ score $(30,41,42,85,88,94,95,99)$, two trials for SCQ score $(44,83)$, one trial for SRS-II score (39), one other trial for PEP-3 score of language and motor skills (92), and one trial for ASI score (86). In six uncontrolled and three controlled trials, patients had another concomitant comorbidity or disorder alongside their main disorder, ASD. Examples include some trials that had patients with a diagnosis of $\operatorname{ADHD}(42,44)$, some other trials that included patients with phobia $(83,89)$, and finally other trials in which some patients had an intellectual disability or language disorder (40, 41, 86, 92, 93).

$\mathrm{AR}$ and VR were integrated into training paradigms through various tools and platforms in the 35 identified trials. From 
these trials, five opted for $\mathrm{AR}$ and 30 for $\mathrm{VR}$ to deliver their intervention. They implemented most of these AR-based programs through smartphone, tablet, or desktop applications and platforms to augment three-dimensional (3D) visual features to the more simplistic features conventionally used in the training paradigms, making them more appealing and engaging for children with $\operatorname{ASD}(29,43,85,98)$. There was one trial that used Google smart glasses equipped with blink sensors, gyroscope, camera, and display screen (44). In this trial, positive feedback was displayed on the screen whenever the participant could successfully gaze at the instructor's face and detect his/her emotion. Alternatively, most of the VR-based interventions were designed on immersive $3 \mathrm{D}$ VE settings in which audiovisual scenes were presented on the walls and ceiling of a room where the participant could fit in different characters in realistic social scenarios. There were also trials that training was based on a particular virtual agent that the participant could play and interact with it. Some VR interventions were also planned on desktop computers using commercial VR software and some of them on HMD devices, which are recently being more and more available and gaining popularity.

More details of included studies for uncontrolled and controlled trials are provided in Tables 1, 2, respectively.

\section{Meta-Analysis}

In the 35 trials entailed in our meta-analysis, ES Hedges $g$ were computed for 167 total number of outcome measures, in which 45 pertained to controlled and 122 pertained to uncontrolled trials. As ESs in two groups were significantly different from each other ( $p=0.0003$, unpaired $t$-test), we were not allowed to combine them into one group, and so we have done all further analyses separately for each of them.

To compute the study-level ESs, its variance, and also between-study variance, we followed the procedure described by Hedges et al. (102). Based on this procedure, an estimate of within-study correlation $(\rho)$ is needed to compute other statistics. As this estimate could not be extracted from most of our included studies, we ran a sensitivity analysis by choosing various values for $\rho$ ranging from 0 to 1 and then computed other statistics based on the chosen value. The results of sensitivity analysis are given in Supplementary Table 1. By this analysis, we inferred that the value study-level computed ES estimates are sensitive to the choice of $\rho$, but it was an ascending function of $\rho$ (the larger the $\rho$, the larger the estimated study-level ESs). Therefore, to avoid any overestimation in computing summary ESs, we fixed the value of $\rho$ at 0 and performed the analysis with this value. It is noteworthy to say that the procedure that we applied in this study is the most parsimonious one avoiding any overstatement of results, but in a realistic situation, the observed effectiveness might be larger as assuming the existence of some level of correlation between study outcomes seems to be a rational assumption. For each trial, we first computed studylevel ESs for all of the outcomes irrespective of their category applying RVE procedure, which gave us overall ES of each study. Then, we repeated this procedure on estimated ESs of outcomes in each category of each study to obtain category-based ESs of that study.

\section{Overall Effectiveness of VR Training}

In the first step, we computed the overall ES for each trial. For nine controlled trials, summary effect size was at medium range $\left(g=0.45, \mathrm{SE}_{g}=0.25\right)$ with moderate heterogeneity $\left(I^{2}=49.5 \%\right.$ and $\left.\tau^{2}=0.055\right)$. Excluding one of the potential outliers with much larger ES ( $g=1.8$ ) (97) led to a bit smaller summary ES, but it was still at the medium range of effectiveness $\left(g=0.38, \mathrm{SE}_{g}\right.$ $=0.2$ ) (Figure 2). For the 26 uncontrolled trials, a large positive ES was found $\left(g=0.74, \mathrm{SE}_{g}=0.17\right)$ with low heterogeneity $\left(I^{2}=\right.$ $2.5 \%$ and $\left.\tau^{2}=0.11\right)$. Excluding one of the potential outliers with extremely large ES $(g=4.8)$ (85) led to similar results $(g=0.736$, $\mathrm{SE}_{g}=0.17$ ) (Figure 3).

We have interpreted the results for overall effectiveness of studies with random-effects model of meta-analysis relying more on controlled trials because of their more robust experimental design.

\section{Skill-Based Effectiveness of VR Training}

Further, ESs were computed for each skill category (defined in Methods). In controlled trials, SCS was addressed in five trials, ERS in three trials, DLS in two trials, and CS in one trial. The effectiveness of VR training was weak for SCS $(g=0.2$, $\left.\mathrm{SE}_{g}=0.23, \tau^{2}=0.03\right)$, weak to moderate for ERS $(g=0.34$, $\left.\mathrm{SE}_{g}=0.06, \tau^{2}=0.02\right)$, and again very strong in DLS $(g=$ 1.37, $\mathrm{SE}_{g}=0.18, \tau^{2}=1.12$ ). The single trial that addressed CS revealed weak to moderate effectiveness $\left(g=0.37, \mathrm{SE}_{g}=\right.$ 0.002 ). Regarding heterogeneity in these estimated summary ESs, a considerably large amount of between-study variance $\left(\tau^{2}\right)$ was observed in DLS category in both design groups, but it was relatively small for SCS, ERS, and CS, and it was small for ERS (compare the $\tau^{2}$ values presented above) (Figure 4).

In uncontrolled trials, SCS had been addressed in 11 trials, ERS in 10 trials, DLS in nine trials and CS in seven trials. VR training led to medium to strong effectiveness in SCS $(g=0.68$, $\left.\mathrm{SE}_{g}=0.08, \tau^{2}=0.13\right)$, medium effectiveness in ERS $(g=0.46$, $\left.\mathrm{SE}_{g}=0.05, \tau^{2}=0.07\right)$, strong effectiveness in DLS $\left(g=1.16, \mathrm{SE}_{g}\right.$ $\left.=0.09, \tau^{2}=0.48\right)$, and medium effectiveness in CS $(g=0.45$, $\mathrm{SE}_{g}=0.02, \tau^{2}=0.03$ ) (Figure 5). Thus, while in other skills we observe promising effectiveness, the DLS is proven to be the most affected area as its strong effectiveness was consistent among both controlled and uncontrolled trials.

\section{Analysis of Confounding Factors}

As a sizable number of trials had not used any legitimate criteria for screening the participants undergoing intervention (e.g., IQ score, social responsiveness score, disease severity, etc.), we recomputed overall ESs for those trials that screened their population applying this kind of criteria to see how much our results would be biased by this potential confounding factor. In the population screened trials, the results for controlled trials were $g=0.25, \mathrm{SE}_{g}=0.1, k=7$, and $\tau^{2}=0.01$ and for uncontrolled trials were $g=0.72, \mathrm{SE}_{g}=0.05, k=12$, and $\tau^{2}=0.04$ and. These results in unscreened trials were $g=0.8$, $\mathrm{SE}_{g}=0.05, k=2$, and $\tau^{2}=0.35$ for controlled and $g=0.73$, $\mathrm{SE}_{g}=0.2, k=14$, and $\tau^{2}=0.18$ for uncontrolled trials.

Comparing these ESs among two screen groups, the results were not meaningfully different from each other in 
TABLE 1 | Characteristics of included studies, uncontrolled trials.

\begin{tabular}{|c|c|c|c|c|c|c|c|c|c|c|c|}
\hline \multirow[t]{2}{*}{ References } & \multirow{2}{*}{$\frac{\text { Age }}{\text { Mean (SD) }}$} & \multirow[t]{2}{*}{$N$} & \multirow[t]{2}{*}{ Type } & \multirow[t]{2}{*}{ Methodic details } & \multirow{2}{*}{$\begin{array}{l}\text { Application } \\
\text { details }\end{array}$} & \multirow[t]{2}{*}{ Study purpose } & \multicolumn{5}{|c|}{ Outcome measure } \\
\hline & & & & & & & Name & Details & Skill & $g$ & $\mathrm{SE}_{g}$ \\
\hline \multirow[t]{5}{*}{ Bai et al. (98) } & \multirow[t]{5}{*}{6.8} & \multirow[t]{5}{*}{12} & \multirow[t]{5}{*}{ AR } & \multirow{5}{*}{$\begin{array}{l}\text { Playing with } \\
\text { augmented toys in } \\
\text { mirror AR display }\end{array}$} & \multirow[t]{5}{*}{$\mathrm{N} / \mathrm{S}$} & \multirow{5}{*}{$\begin{array}{l}\text { Improve and learn } \\
\text { pretend play and } \\
\text { representation of } \\
\text { pretense }\end{array}$} & Pretend play frequency & \multirow[t]{5}{*}{ Play Observation Scale } & \multirow[t]{4}{*}{ CS } & 0.7 & 0.42 \\
\hline & & & & & & & Pretend play duration & & & 0.94 & 0.47 \\
\hline & & & & & & & Constructive play frequency & & & 1.42 & 0.59 \\
\hline & & & & & & & Constructive play duration & & & 1.01 & 0.49 \\
\hline & & & & & & & & & Overall & 1.02 & 0.6 \\
\hline \multirow{7}{*}{$\begin{array}{l}\text { Bernardini et al. } \\
(100)\end{array}$} & \multirow[t]{7}{*}{$\mathrm{N} / \mathrm{M}$} & \multirow[t]{7}{*}{19} & \multirow[t]{7}{*}{ VR } & \multirow{7}{*}{$\begin{array}{l}\text { Playing game with } \\
\text { VA }\end{array}$} & \multirow{7}{*}{$\begin{array}{l}\text { Several } 10 \text { - to } \\
20 \text {-min sessions } \\
\text { in a week for } 8 \\
\text { weeks }\end{array}$} & \multirow{7}{*}{$\begin{array}{l}\text { Help children } \\
\text { acquire social } \\
\text { communication } \\
\text { skills }\end{array}$} & Response to social partner & \multirow{7}{*}{$\begin{array}{l}\text { SAP to assess } \\
\text { socioemotional abilities of } \\
\text { autistics }\end{array}$} & \multirow[t]{6}{*}{ SCS } & 0.07 & 0.42 \\
\hline & & & & & & & Initiation to social partner & & & 0.02 & 0.42 \\
\hline & & & & & & & Social behavior & & & 0.02 & 0.42 \\
\hline & & & & & & & $\begin{array}{l}\text { Sequences of social } \\
\text { behaviors }\end{array}$ & & & -0.25 & 0.43 \\
\hline & & & & & & & $\begin{array}{l}\text { Speech toward social } \\
\text { partner }\end{array}$ & & & 0.06 & 0.42 \\
\hline & & & & & & & Missed opportunities & & & 0.81 & 0.54 \\
\hline & & & & & & & & & Overall & 0.12 & 0.55 \\
\hline \multirow[t]{2}{*}{ Chen et al. (85) } & \multirow[t]{2}{*}{11.5} & \multirow[t]{2}{*}{6} & $\mathrm{AR}$ & ARVMS & Seven sessions & $\begin{array}{l}\text { Facial expressions } \\
\text { and emotions of } \\
\text { others in social } \\
\text { situations }\end{array}$ & Performance & Instructor assessment & ERS & 4.81 & 2.94 \\
\hline & & & & & & & & & Overall & 4.81 & 2.96 \\
\hline Didehbani et al. & $11.4(2.7)$ & 30 & VR & Social scenarios in & Ten 1-h sessions & Enhance emotion & NEPSY-2 affect recognition & Facial affect recognition & ERS & 0.66 & 0.23 \\
\hline (42) & & & & $\begin{array}{l}\text { customized Second } \\
\text { Life }^{T M} \text { VE }\end{array}$ & & & EKMAN 60 & $\begin{array}{l}\text { Recognition of basic } \\
\text { emotions }\end{array}$ & & 0.46 & 0.31 \\
\hline & & & & & & $\begin{array}{l}\text { attention and } \\
\text { executive function }\end{array}$ & Triangle total & $\begin{array}{l}\text { Understanding of social } \\
\text { intentionality }\end{array}$ & SCS & 0.38 & 0.22 \\
\hline & & & & & & & Triangle intentionality & & & 0.45 & 0.23 \\
\hline & & & & & & & Fluid reasoning & $\begin{array}{l}\text { Selective attention and } \\
\text { concentration }\end{array}$ & CS & 0.52 & 0.27 \\
\hline & & & & & & & & & Overall & 0.44 & 0.41 \\
\hline Ip et al. (99) & 8.7 & 33 & VR & $\begin{array}{l}\text { School-related } \\
\text { social scenarios in }\end{array}$ & 28 sessions & $\begin{array}{l}\text { Enhance social } \\
\text { skills and coping }\end{array}$ & Eyes test & Emotion recognition & ERS & 0.53 & 0.29 \\
\hline & & & & four-sided CAVE & & skills while avoid & Affective expression & & & 0.68 & 0.31 \\
\hline & & & & & & unnecessary & Social reciprocity & Social reciprocity & SCS & 0.6 & 0.3 \\
\hline & & & & & & & PEP-3 overall & $\begin{array}{l}\text { Social functioning and } \\
\text { communication }\end{array}$ & & 0.76 & 0.32 \\
\hline & & & & & & & & & Overall & 0.64 & 0.45 \\
\hline $\begin{array}{l}\text { Josman et al. } \\
(40)\end{array}$ & $13.2(3)$ & 6 & VR & $\begin{array}{l}\text { Street crossing in VE } \\
\text { computer program }\end{array}$ & $\begin{array}{l}\text { Eight 10- to } \\
\text { 30-min sessions }\end{array}$ & $\begin{array}{l}\text { Teach street } \\
\text { crossing skill }\end{array}$ & $\begin{array}{l}\mathrm{N} \text { of left looking at first } \\
\text { crosswalk }\end{array}$ & $\begin{array}{l}\text { Participant performance in } \\
\text { VR software }\end{array}$ & DLS & 1.72 & 1.16 \\
\hline & & & & & & & $\begin{array}{l}\mathrm{N} \text { of right looking at first } \\
\text { crosswalk }\end{array}$ & & & 0.58 & 0.63 \\
\hline
\end{tabular}


TABLE 1 | Continued

\begin{tabular}{|c|c|c|c|c|c|c|c|c|c|c|c|}
\hline \multirow[t]{2}{*}{ References } & \multirow{2}{*}{$\frac{\text { Age }}{\text { Mean (SD) }}$} & \multirow[t]{2}{*}{$N$} & \multirow[t]{2}{*}{ Type } & \multirow[t]{2}{*}{ Methodic details } & \multirow{2}{*}{$\begin{array}{l}\text { Application } \\
\text { details }\end{array}$} & \multirow[t]{2}{*}{ Study purpose } & \multicolumn{5}{|c|}{ Outcome measure } \\
\hline & & & & & & & Name & Details & Skill & $g$ & $\mathrm{SE}_{g}$ \\
\hline & & & & & & & $\begin{array}{l}\mathrm{N} \text { of left looking at second } \\
\text { crosswalk }\end{array}$ & & & 0 & 0.53 \\
\hline & & & & & & & $\begin{array}{l}\mathrm{N} \text { of right looking at second } \\
\text { crosswalk }\end{array}$ & & & 0.37 & 0.57 \\
\hline & & & & & & & $\begin{array}{l}\text { total } \mathrm{N} \text { of left looking } \\
\text { crossing the road }\end{array}$ & & & 1.4 & 0.99 \\
\hline & & & & & & & $\begin{array}{l}\text { total } \mathrm{N} \text { of right looking } \\
\text { crossing the road }\end{array}$ & & & 0.19 & 0.54 \\
\hline & & & & & & & $\begin{array}{l}\text { N looked left at crosswalk } \\
\text { with traffic light }\end{array}$ & & & 0.18 & 0.54 \\
\hline & & & & & & & $\begin{array}{l}\mathrm{N} \text { looked right at crosswalk } \\
\text { with traffic light }\end{array}$ & & & 0.45 & 0.59 \\
\hline & & & & & & & $\begin{array}{l}\mathrm{N} \text { of accidents at the } \\
\text { crosswalk with traffic light }\end{array}$ & & & 0.75 & 0.69 \\
\hline & & & & & & & & & Overall & 0.63 & 0.8 \\
\hline \multirow{8}{*}{$\begin{array}{l}\text { Kandalaft et al. } \\
\text { (87) }\end{array}$} & \multirow[t]{8}{*}{$21.2(2.7)$} & \multirow[t]{8}{*}{8} & \multirow[t]{8}{*}{ VR } & \multirow{8}{*}{$\begin{array}{l}\text { Interacting with VA in } \\
\text { second Life }{ }^{\mathrm{TM}} \\
\text { software }\end{array}$} & \multirow[t]{8}{*}{10 sessions } & \multirow{8}{*}{$\begin{array}{l}\text { Enhancing social } \\
\text { skills, social } \\
\text { cognition, and } \\
\text { social functioning }\end{array}$} & SP-total & \multirow{4}{*}{$\begin{array}{l}\text { Verbal and non-verbal } \\
\text { emotion recognition by } \\
\text { ACS-SP }\end{array}$} & \multirow[t]{4}{*}{ ERS } & 0.89 & 0.56 \\
\hline & & & & & & & SP-affect & & & 0.39 & 0.45 \\
\hline & & & & & & & SP-prosody & & & 1.03 & 0.59 \\
\hline & & & & & & & SP-pair & & & 0.59 & 0.48 \\
\hline & & & & & & & EKMAN 60 & \multirow[t]{2}{*}{ Theory of mind (ToM) } & \multirow[t]{3}{*}{ scs } & 1.25 & 0.66 \\
\hline & & & & & & & Triangle & & & 1.08 & 0.61 \\
\hline & & & & & & & SSPA & Conversation skills & & 0.32 & 0.44 \\
\hline & & & & & & & & & Overall & 0.79 & 0.64 \\
\hline \multirow[t]{6}{*}{ Ke et al. (95) } & \multirow[t]{6}{*}{$\mathrm{N} / \mathrm{M}$} & \multirow[t]{6}{*}{8} & \multirow[t]{6}{*}{ VR } & \multirow{6}{*}{$\begin{array}{l}\text { 3D virtual world } \\
\text { designed by } \\
\text { OpenSimulator }\end{array}$} & \multirow{6}{*}{$\begin{array}{l}\text { Average of } \\
20.22 \mathrm{~h} \text {, over } \\
16-31 \text { sessions }\end{array}$} & \multirow{6}{*}{$\begin{array}{l}\text { Enhance social } \\
\text { skills }\end{array}$} & Responding & \multirow{6}{*}{$\begin{array}{l}\text { Performance evaluated by } \\
\text { instructors }\end{array}$} & \multirow[t]{5}{*}{ SCS } & 0.02 & 0.42 \\
\hline & & & & & & & Initiation & & & 1.26 & 0.67 \\
\hline & & & & & & & Negotiation & & & 1.61 & 0.78 \\
\hline & & & & & & & Self-identification & & & 0.83 & 0.54 \\
\hline & & & & & & & Cognitive flexibility & & & 2.09 & 0.96 \\
\hline & & & & & & & & & Overall & 1.16 & 0.77 \\
\hline \multirow[t]{2}{*}{$\begin{array}{l}\text { Kurniawan et al. } \\
\text { (29) }\end{array}$} & $\mathrm{N} / \mathrm{M}$ & 12 & $A R$ & PECS-AR & $N / S$ & $\begin{array}{l}\text { communication } \\
\text { ability }\end{array}$ & Communication ability score & Teacher's assessment & SCS & 1.26 & 0.47 \\
\hline & & & & & & & & & Overall & 1.26 & 0.58 \\
\hline $\begin{array}{l}\text { Lamash et al. } \\
\text { (36) }\end{array}$ & $14.6(1.8)$ & 33 & VR & $\begin{array}{l}\text { Shopping training in } \\
\text { VAP-S software }\end{array}$ & Five sessions & $\begin{array}{l}\text { Shopping skills, } \\
\text { executive cognitive }\end{array}$ & $\begin{array}{l}\text { WebNeuro attention } \\
\text { component }\end{array}$ & $\begin{array}{l}\text { Evaluation of cognitive and } \\
\text { meta-cognitive functis }\end{array}$ & CS & 0.58 & 0.2 \\
\hline & & & & & & $\begin{array}{l}\text { and metacognitive } \\
\text { skills }\end{array}$ & $\begin{array}{l}\text { WebNeuro executive } \\
\text { function component }\end{array}$ & & & 0.58 & 0.2 \\
\hline & & & & & & & $\begin{array}{l}\text { WebNeuro verbal } \\
\text { component }\end{array}$ & & & -0.38 & 0.19 \\
\hline
\end{tabular}




\begin{tabular}{|c|c|c|c|c|c|c|c|c|c|c|c|}
\hline \multirow[t]{2}{*}{ References } & \multirow{2}{*}{$\frac{\text { Age }}{\overline{\text { Mean (SD) }}}$} & \multirow[t]{2}{*}{$N$} & \multirow[t]{2}{*}{ Type } & \multirow[t]{2}{*}{ Methodic details } & \multirow{2}{*}{$\begin{array}{l}\text { Application } \\
\text { details }\end{array}$} & \multirow[t]{2}{*}{ Study purpose } & \multicolumn{5}{|c|}{ Outcome measure } \\
\hline & & & & & & & Name & Details & Skill & $g$ & $\mathrm{SE}_{g}$ \\
\hline & & & & & & & TOGGS accuracy & TOGGS, performance in & DLS & 1.5 & 0.27 \\
\hline & & & & & & & TOGGS time & shopping & & 0.62 & 0.2 \\
\hline & & & & & & & TOGGS redundancy & & & 0.93 & 0.22 \\
\hline & & & & & & & TOGGS strategy usage & & & 1.85 & 0.31 \\
\hline & & & & & & & & & Overall & 0.81 & 0.4 \\
\hline \multirow[t]{3}{*}{ Manju et al. (30) } & \multirow[t]{3}{*}{$4.6(0.9)$} & \multirow[t]{3}{*}{5} & \multirow[t]{3}{*}{ VR } & \multirow{3}{*}{$\begin{array}{l}\text { VE with scenes } \\
\text { presented on wall }\end{array}$} & \multirow[t]{3}{*}{$\mathrm{N} / \mathrm{S}$} & \multirow{3}{*}{$\begin{array}{l}\text { Social skills and } \\
\text { attention }\end{array}$} & Likert score & Attention grasping & CS & 2.39 & 2.07 \\
\hline & & & & & & & Likert score & Social interaction & SCS & 1.6 & 1.47 \\
\hline & & & & & & & & & Overall & 1.99 & 1.82 \\
\hline \multirow[t]{3}{*}{$\begin{array}{l}\text { Maskey et al. } \\
\text { (89) }\end{array}$} & \multirow[t]{3}{*}{$11.2(2)$} & \multirow[t]{3}{*}{9} & \multirow[t]{3}{*}{ VR } & \multirow[t]{3}{*}{$\begin{array}{l}\text { Exposure to fearful } \\
\text { stimuli in VE }\end{array}$} & \multirow[t]{3}{*}{$\begin{array}{l}\text { Four 20- to } \\
\text { 30-min sessions }\end{array}$} & \multirow{3}{*}{$\begin{array}{l}\text { Reduction or } \\
\text { treating specific } \\
\text { phobia }\end{array}$} & SCAS-P & $\begin{array}{l}\text { Children's Anxiety Scale } \\
\text { parent score }\end{array}$ & ES & 0.62 & 0.46 \\
\hline & & & & & & & SCAS-C & $\begin{array}{l}\text { Children's Anxiety Scale } \\
\text { child score }\end{array}$ & & 0.66 & 0.45 \\
\hline & & & & & & & & & Overall & 0.64 & 0.56 \\
\hline \multirow[t]{8}{*}{$\begin{array}{l}\text { Maskey et al. } \\
\text { (83) }\end{array}$} & \multirow[t]{8}{*}{29.8} & \multirow[t]{8}{*}{8} & \multirow[t]{8}{*}{ VR } & \multirow[t]{8}{*}{ Blue VR room } & $\begin{array}{l}\text { Four 20-min } \\
\text { sessions of } \\
\text { graded exposure }\end{array}$ & $\begin{array}{l}\text { Treating phobia } \\
\text { and anxiety }\end{array}$ & Anxiety BAI score & BAl & DLS & 0.03 & 0.42 \\
\hline & & & & & & & Anxiety GAD score & GAD-7 & & -0.04 & 0.42 \\
\hline & & & & & & & Depression score & PHQ-9 & & 0.32 & 0.44 \\
\hline & & & & & & & Quality of life (QoL) physical & $\begin{array}{l}\text { WHOQOL-BREF } \\
\text { questionnaire }\end{array}$ & & -0.47 & 0.46 \\
\hline & & & & & & & QoL psychological & Addresses QoL & & -0.03 & 0.42 \\
\hline & & & & & & & QoL social & & & -1.2 & 0.66 \\
\hline & & & & & & & QoL environmental & & & 0.2 & 0.42 \\
\hline & & & & & & & & & Overall & -0.17 & 0.58 \\
\hline Miller et al. (101) & 5.2 & 5 & $\mathrm{VR}$ & HMD, Google & One session per & Improve air travel & Parent score & 5-Point Likert score & DLS & 0.98 & 1.03 \\
\hline & & & & cardboard & week for 3 & skills & Researcher score & & & 1.1 & 1.11 \\
\hline & & & & & weeks & & & & Overall & 1.04 & 1.12 \\
\hline Milne et al. (96) & 10.5 & 14 & VR & Interacting with VA & $\mathrm{N} / \mathrm{S}$ & Teaching social & Conservation skills & Performance in social & DLS & 0.67 & 0.33 \\
\hline & & & & & & skills and how to & Dealing with bully skills & scenarios measured by & & 1.09 & 0.39 \\
\hline & & & & & & cope with bullying & & evaluators scoring & Overall & 0.88 & 0.49 \\
\hline Nubia et al. (43) & 6 & 5 & AR & $\begin{array}{l}\text { Pictogram } \\
\text { recognition task }\end{array}$ & N/S & $\begin{array}{l}\text { Improve attention } \\
\text { process and } \\
\text { appearance of }\end{array}$ & Attention process & $\begin{array}{l}\text { No. of children successfully } \\
\text { finished the attention task }\end{array}$ & CS & 0.53 & 0.3 \\
\hline & & & & & & verbal language & Emergence of language & & & 0.55 & 0.31 \\
\hline & & & & & & & & & Overall & 0.54 & 0.45 \\
\hline Ross et al. (37) & 18 & 46 & VR & Driving simulation in & $8-12$ sessions & Improve attitude & DAS-PR positive attitude & Driving Attitude & DLS & 1.74 & 0.25 \\
\hline & & & & & & toward driving & DAS-PR negative attitude & Scale-Parent Report & & 1.07 & 0.19 \\
\hline & & & & & & & & & Overall & 1.41 & 0.4 \\
\hline
\end{tabular}

Anxiety GAD score

epression score

QoL psychological

QoL social

Parent score

week for 3 skills Researcher score

Teaching socia

skills and how to

prove attention

process and

appearance of

Improve attitude

toward driving 
TABLE 1 | Continued

\begin{tabular}{|c|c|c|c|c|c|c|c|c|c|c|c|}
\hline \multirow[t]{2}{*}{ References } & \multirow{2}{*}{$\frac{\text { Age }}{\text { Mean (SD) }}$} & \multirow[t]{2}{*}{$N$} & \multirow[t]{2}{*}{ Type } & \multirow[t]{2}{*}{ Methodic details } & \multirow{2}{*}{$\begin{array}{l}\text { Application } \\
\text { details }\end{array}$} & \multirow[t]{2}{*}{ Study purpose } & \multicolumn{5}{|c|}{ Outcome measure } \\
\hline & & & & & & & Name & Details & Skill & $g$ & $\mathrm{SE}_{g}$ \\
\hline \multirow[t]{7}{*}{ Saiano et al. (41) } & \multirow[t]{7}{*}{$24(10)$} & \multirow[t]{7}{*}{6} & \multirow[t]{7}{*}{ VR } & \multirow{7}{*}{$\begin{array}{l}\text { Street crossing and } \\
\text { path following in VE } \\
\text { representing a city }\end{array}$} & \multirow{7}{*}{$\begin{array}{l}\text { Ten } 45 \text {-min } \\
\text { sessions }\end{array}$} & \multirow{7}{*}{$\begin{array}{l}\text { Teaching of street } \\
\text { crossing and path } \\
\text { following skills }\end{array}$} & Caregiver score & Likert score questionnaire & DLS & 1.85 & 1.23 \\
\hline & & & & & & & Parent score & & & 0.92 & 0.76 \\
\hline & & & & & & & Speed & $\begin{array}{l}\text { Subject performance in city } \\
\text { surveying and street } \\
\text { crossing }\end{array}$ & & 1.71 & 1.15 \\
\hline & & & & & & & Composite index & & & 0.45 & 0.59 \\
\hline & & & & & & & Figural distance & & & 0.75 & 0.69 \\
\hline & & & & & & & Path length taken & & & 0.48 & 0.6 \\
\hline & & & & & & & & & Overall & 1.03 & 0.94 \\
\hline \multirow[t]{5}{*}{$\begin{array}{l}\text { Simoes et al. } \\
\text { (93) }\end{array}$} & \multirow[t]{5}{*}{$18.8(4.5)$} & \multirow[t]{5}{*}{6} & \multirow[t]{5}{*}{ VR } & \multirow{5}{*}{$\begin{array}{l}\text { Street crossing and } \\
\text { bus taking in VE } \\
\text { presented by HMD }\end{array}$} & \multirow[t]{5}{*}{$\begin{array}{l}\text { Three } 20 \text { to } \\
40 \text {-min sessions }\end{array}$} & \multirow{5}{*}{$\begin{array}{l}\text { Teaching } \\
\text { bus-taking } \\
\text { routines and } \\
\text { effectively using } \\
\text { bus for } \\
\text { transformation }\end{array}$} & $\begin{array}{l}\text { Action accuracy } \\
\text { Debriefing accuracy }\end{array}$ & Performance in bus taking & DLS & $\begin{array}{l}1.1 \\
1.8\end{array}$ & $\begin{array}{c}0.5 \\
0.69\end{array}$ \\
\hline & & & & & & & Global EDA & Stress level & ERS & 0.66 & 0.66 \\
\hline & & & & & & & Bus EDA & & & 0.81 & 0.72 \\
\hline & & & & & & & Streets EDA & & & 0.51 & 0.61 \\
\hline & & & & & & & & & Overall & 0.98 & 0.72 \\
\hline \multirow{12}{*}{$\begin{array}{l}\text { Stichter et al. } \\
\text { (88) }\end{array}$} & \multirow[t]{12}{*}{$12.6(0.7)$} & \multirow[t]{12}{*}{11} & \multirow[t]{12}{*}{ VR } & \multirow{12}{*}{$\begin{array}{l}\text { Social competence } \\
\text { tasks in } \\
\text { computer-generated } \\
\text { 3D VE }\end{array}$} & \multirow{12}{*}{$\begin{array}{l}31 \text { sessions over } \\
\text { a 4-month } \\
\text { period }\end{array}$} & \multirow{12}{*}{$\begin{array}{l}\text { Enhance social } \\
\text { competence }\end{array}$} & SRS total parent score & Social Responsiveness & SCS & 1.04 & 0.46 \\
\hline & & & & & & & $\begin{array}{l}\text { SRS social awareness } \\
\text { parent score }\end{array}$ & Scale & & 0.47 & 0.36 \\
\hline & & & & & & & $\begin{array}{l}\text { SRS social cognition parent } \\
\text { score }\end{array}$ & & & 1.15 & 0.48 \\
\hline & & & & & & & $\begin{array}{l}\text { SRS social communication } \\
\text { parent score }\end{array}$ & & & 1.26 & 0.51 \\
\hline & & & & & & & $\begin{array}{l}\text { SRS social motivation } \\
\text { parent score }\end{array}$ & & & 0.75 & 0.41 \\
\hline & & & & & & & SRS total teacher score & & & 0.53 & 0.37 \\
\hline & & & & & & & $\begin{array}{l}\text { SRS social awareness } \\
\text { teacher score }\end{array}$ & & & 0.34 & 0.35 \\
\hline & & & & & & & $\begin{array}{l}\text { SRS social cognition } \\
\text { teacher score }\end{array}$ & & & -0.12 & 0.34 \\
\hline & & & & & & & $\begin{array}{l}\text { SRS social communication } \\
\text { teacher score }\end{array}$ & & & 0.6 & 0.38 \\
\hline & & & & & & & $\begin{array}{l}\text { SRS social motivation } \\
\text { teacher score }\end{array}$ & & & 0.34 & 0.35 \\
\hline & & & & & & & $\begin{array}{l}\text { BRIEF global executive } \\
\text { parent score }\end{array}$ & $\begin{array}{l}\text { Behavior Rating Inventory of } \\
\text { Executive Function }\end{array}$ & CS & 0.68 & 0.39 \\
\hline & & & & & & & $\begin{array}{l}\text { BRIEF behavioral regulation } \\
\text { parent score }\end{array}$ & & & 0.45 & 0.36 \\
\hline
\end{tabular}


TABLE 1 | Continued

\begin{tabular}{|c|c|c|c|c|c|c|c|c|c|c|c|}
\hline \multirow[t]{2}{*}{ References } & \multirow{2}{*}{$\frac{\text { Age }}{\text { Mean (SD) }}$} & \multirow[t]{2}{*}{$N$} & \multirow[t]{2}{*}{ Type } & \multirow[t]{2}{*}{ Methodic details } & \multirow{2}{*}{$\begin{array}{l}\text { Application } \\
\text { details }\end{array}$} & \multirow[t]{2}{*}{ Study purpose } & \multicolumn{5}{|c|}{ Outcome measure } \\
\hline & & & & & & & Name & Details & Skill & $g$ & $\mathrm{SE}_{g}$ \\
\hline & & & & & & & $\begin{array}{l}\text { BRIEF metacognition parent } \\
\text { score }\end{array}$ & & & 0.64 & 0.39 \\
\hline & & & & & & & $\begin{array}{l}\text { BRIEF global executive } \\
\text { teacher score }\end{array}$ & & & 0.5 & 0.37 \\
\hline & & & & & & & $\begin{array}{l}\text { BRIEF behavioral regulation } \\
\text { teacher score }\end{array}$ & & & 0.14 & 0.34 \\
\hline & & & & & & & $\begin{array}{l}\text { BRIEF metacognition } \\
\text { teacher score }\end{array}$ & & & 0.33 & 0.35 \\
\hline & & & & & & & Reading in mind's eye & Student performance & ERS & 0.17 & 0.34 \\
\hline & & & & & & & Faux pas stories & & & -0.35 & 0.35 \\
\hline & & & & & & & Strange stories & & & 0.25 & 0.35 \\
\hline & & & & & & & DANVA & $\begin{array}{l}\text { Child facial expression } \\
\text { analysis }\end{array}$ & & 0.44 & 0.36 \\
\hline & & & & & & & $\begin{array}{l}\text { Trail making: number letter } \\
\text { switching }\end{array}$ & $\begin{array}{l}\text { D-KEFS Delis-Kaplan } \\
\text { executive functioning } \\
\text { system }\end{array}$ & CS & 0.17 & 0.34 \\
\hline & & & & & & & $\begin{array}{l}\text { Design fluency: switching } \\
\text { designs }\end{array}$ & & & 0.62 & 0.38 \\
\hline & & & & & & & $\begin{array}{l}\text { Design fluency: total correct } \\
\text { designs }\end{array}$ & & & 1.06 & 0.46 \\
\hline & & & & & & & $\begin{array}{l}\text { Color-word interface: } \\
\text { inhibition task }\end{array}$ & & & -0.03 & 0.34 \\
\hline & & & & & & & $\begin{array}{l}\text { Color-word interface: } \\
\text { inhibit/switch }\end{array}$ & & & 0.16 & 0.34 \\
\hline & & & & & & & $\begin{array}{l}\text { CPT-2 overall omission } \\
\text { errors }\end{array}$ & $\begin{array}{l}\text { Continuous performance } \\
\text { test-II (CPT-II) }\end{array}$ & & 0.09 & 0.34 \\
\hline & & & & & & & $\begin{array}{l}\text { CPT-2 overall commission } \\
\text { errors }\end{array}$ & & & 0.15 & 0.34 \\
\hline & & & & & & & & & Overall & 0.44 & 0.5 \\
\hline \multirow[t]{2}{*}{$\begin{array}{l}\text { Vahabzade et al. } \\
\text { (44) }\end{array}$} & $15(3.4)$ & 8 & AR & $\begin{array}{l}\text { Maintain gaze } \\
\text { toward faces by AR } \\
\text { smart glasses }\end{array}$ & One session & $\begin{array}{l}\text { Improving gaze } \\
\text { duration to faces } \\
\text { and reducing } \\
\text { ADHD symptoms }\end{array}$ & $\mathrm{ABC}-\mathrm{H}$ score & $\begin{array}{l}\text { Measure of ADHD } \\
\text { symptoms }\end{array}$ & CS & 0.72 & 0.51 \\
\hline & & & & & & & & & Overall & 0.72 & 0.61 \\
\hline \multirow[t]{2}{*}{ Wade et al. (38) } & $15.9(1.3)$ & 6 & VR & $\begin{array}{l}\text { Driving simulation in } \\
\text { VE }\end{array}$ & $\begin{array}{l}\text { Six visits of three } \\
\text { driving sessions } \\
\text { in } 24 \text { trials }\end{array}$ & $\begin{array}{l}\text { Improve safe } \\
\text { driving skills }\end{array}$ & Performance-based failures & Subject's performance & DLS & 1.98 & 1.3 \\
\hline & & & & & & & & & Overall & 1.98 & 1.34 \\
\hline
\end{tabular}


TABLE 1 | Continued

\begin{tabular}{|c|c|c|c|c|c|c|c|c|c|c|c|}
\hline \multirow[t]{2}{*}{ References } & \multirow{2}{*}{$\frac{\text { Age }}{\text { Mean (SD) }}$} & \multirow[t]{2}{*}{$N$} & \multirow[t]{2}{*}{ Type } & \multirow[t]{2}{*}{ Methodic details } & \multirow{2}{*}{$\begin{array}{l}\text { Application } \\
\text { details }\end{array}$} & \multirow[t]{2}{*}{ Study purpose } & \multicolumn{5}{|c|}{ Outcome measure } \\
\hline & & & & & & & Name & Details & Skill & $g$ & $\mathrm{SE}_{g}$ \\
\hline \multirow[t]{3}{*}{ Wade et al. (39) } & $15.3(1.6)$ & 8 & VR & 3D game driving & Six $75-\min$ & Enhancing driving & Duration time & Performance & DLS & 0.73 & 0.51 \\
\hline & & & & simulator & sessions & & No. of failures & & & 1.27 & 0.67 \\
\hline & & & & & & & & & Overall & 1 & 0.7 \\
\hline \multirow[t]{3}{*}{ Yang et al. (94) } & $22.5(3.9)$ & 17 & VR & $\begin{array}{l}\text { VR-SCT computer } \\
\text { program }\end{array}$ & Ten 1 -h sessions & $\begin{array}{l}\text { Emotion } \\
\text { recognition } \\
\text { training and ToM } \\
\text { or sociocognitive }\end{array}$ & $\begin{array}{l}\text { ACS-SP emotion } \\
\text { recognition }\end{array}$ & Social Perception & ERS & 0.89 & 0.56 \\
\hline & & & & & & skills improvement & ToM triangle test & ToM & SCS & 1.08 & 0.61 \\
\hline & & & & & & & & & Overall & 0.99 & 0.67 \\
\hline \multirow[t]{3}{*}{ Yuan et al. (84) } & $9(1.1)$ & 36 & VR & $\begin{array}{l}\text { Social scenarios in } \\
\text { four-sided CAVE }\end{array}$ & One 1-h session & $\begin{array}{l}\text { Train emotional } \\
\text { and social skills }\end{array}$ & PEP-3 affective expressions & $\begin{array}{l}\text { Emotion expression and } \\
\text { regulation }\end{array}$ & ERS & 0.35 & 0.18 \\
\hline & & & & & & & PEP-3 social reciprocity & $\begin{array}{l}\text { Social interaction and } \\
\text { adaptation }\end{array}$ & SCS & 0.64 & 0.19 \\
\hline & & & & & & & & & Overall & 0.5 & 0.38 \\
\hline \multirow[t]{7}{*}{ Zhao et al. (31) } & $12.4(2.6)$ & 12 & VR & Social games in CVE & $\mathrm{N} / \mathrm{S}$ & $\begin{array}{l}\text { Motor skill and } \\
\text { social interaction }\end{array}$ & $\begin{array}{l}\text { Completed pieces }(/ \mathrm{min}) \\
\text { study } 1\end{array}$ & Performance in puzzle game & SCS & 1.07 & 0.83 \\
\hline & & & & & & simultaneously & $\begin{array}{l}\text { Cooperative efficacy \% } \\
\text { study } 1\end{array}$ & & & 0.76 & 0.7 \\
\hline & & & & & & & Total play time (s) study 1 & & & 0.9 & 0.75 \\
\hline & & & & & & & $\begin{array}{l}\text { Word count of ASD } \\
\text { subjects(/min) study } 1\end{array}$ & & & 0.34 & 0.57 \\
\hline & & & & & & & $\begin{array}{l}\text { Back-and-forth } \\
\text { sentences//min) study } 1\end{array}$ & & & -0.65 & 0.66 \\
\hline & & & & & & & Aggregate score study 1 & & & 0.76 & 0.7 \\
\hline & & & & & & & & & Overall & 0.53 & 0.8 \\
\hline
\end{tabular}

ABC-H, Aberrant Behavioral Checklist; ACS, Advanced Clinical Solutions; ADHD, attention-deficit/hyperactivity disorder; AR, augmented reality; ARVMS, Augmented Reality Video Modeling System; BAI, Beck Anxiety Inventory; BRIEF, Behavior Rating Inventory of Executive Function; CAVE, Cave Automatic Virtual Environment; CPT, continuous performance test; CS, cognitive skills; CVE, collaborative virtual environment; DANVA, Diagnostic Analysis of Non-verbal Accuracy; DAS-PR, Driving Attitude Scale-Parent Report; DLS, daily living skills; EDA, electrodermal activity; ERS, emotion regulation and recognition skills; GAD-7, Generalized Anxiety Disorder-7; N, number of participants; N/S, not specified; NEPSY, a developmental NEuroPSYchological assessment; NM, not mentioned; PECS, picture exchange communication system; PEP, psychoeducational profile; PHQ-9, Patient Health Questionnaire-9; SAP, SCERTS assessment protocol; SCAS_C, Spence Children's Anxiety Scale-Child Version; SCAS_P, Spence Children's Anxiety Scale-Parent Version; SCQ, Social Communication Questionnaire; SCS, social and communication skills; SRS, Social Responsiveness Scale; SSPA, Social Skills Performance Assessment; TOGGS, Test of Grocery Shopping Skills; VA, virtual avatar; VE, virtual environment; VR, virtual reality; VR-SCT, virtual reality social cognition training. The bold values represent overall effectiveness for each study. 
TABLE 2 | Characteristics of included studies, controlled trials.

\begin{tabular}{|c|c|c|c|c|c|c|c|c|c|c|c|}
\hline \multirow[t]{2}{*}{ References } & \multirow{2}{*}{$\frac{\text { Age }}{\text { Mean (SD) }}$} & \multicolumn{2}{|c|}{ Number } & \multirow[t]{2}{*}{ Methodic details } & \multirow{2}{*}{$\begin{array}{l}\text { Application } \\
\text { details }\end{array}$} & \multirow[t]{2}{*}{ Study purpose } & \multicolumn{5}{|c|}{ Outcome measure } \\
\hline & & NI & NC & & & & Name & Details & Skill & $g$ & SEg \\
\hline \multirow[t]{3}{*}{ Chen et al. (92) } & $4.9(1.1)$ & 11 & 11 & 3D virtual & Three sessions & Improve speech & Consonants & Rated with linguistis & CS & 0.36 & 0.45 \\
\hline & & & & punctuation tutor & & & Vowels & & & 0.38 & 0.45 \\
\hline & & & & & & & & & Overall & 0.37 & 0.5 \\
\hline \multirow[t]{9}{*}{ Ip et al. (33) } & 13.55 & 36 & 36 & $\begin{array}{l}\text { Social scenarios in } \\
\text { half-CAVE }\end{array}$ & $\begin{array}{l}\text { 28, 30-min } \\
\text { sessions }\end{array}$ & $\begin{array}{l}\text { Improving emotion } \\
\text { recognition, }\end{array}$ & $\begin{array}{l}\text { Faces test } \\
\text { Eyes test }\end{array}$ & Emotion recognition & ERS & $\begin{array}{l}0.26 \\
0.14\end{array}$ & $\begin{array}{l}0.24 \\
0.24\end{array}$ \\
\hline & & & & & & $\begin{array}{l}\text { emotion } \\
\text { expression and }\end{array}$ & PEP-3 affective expressions & $\begin{array}{l}\text { Emotion expression, } \\
\text { regulation, and social }\end{array}$ & & 0.44 & 0.24 \\
\hline & & & & & & $\begin{array}{l}\text { social reciprocity, } \\
\text { social adaptive }\end{array}$ & PEP-3 social reciprocity & reciprocity & & 0.47 & 0.24 \\
\hline & & & & & & & ABAS communication & Social adaptive skills & SCS & 0.13 & 0.24 \\
\hline & & & & & & & ABAS community use & & & -0.64 & 0.25 \\
\hline & & & & & & & ABAS leisure & & & -0.24 & 0.24 \\
\hline & & & & & & & ABAS self-direction & & & -0.48 & 0.24 \\
\hline & & & & & & & ABAS social & & & -0.23 & 0.24 \\
\hline & & & & & & & & & Overall & -0.05 & 0.34 \\
\hline \multirow[t]{2}{*}{$\begin{array}{l}\text { Lamash et al. } \\
\text { (36) }\end{array}$} & $\begin{array}{l}14.58 \\
(1.77)\end{array}$ & 33 & 23 & $\begin{array}{l}\text { Shopping training in } \\
\text { VAP-S software }\end{array}$ & Five sessions & $\begin{array}{l}\text { Improving } \\
\text { shopping skills }\end{array}$ & TOGGS accuracy & Performance in shopping & DLS & 1.02 & 0.29 \\
\hline & & & & & & & & & Overall & 1.02 & 0.38 \\
\hline \multirow[t]{10}{*}{$\begin{array}{l}\text { Maskey et al. } \\
\text { (83) }\end{array}$} & $10.8(2)$ & 16 & 16 & Blue room VR & Four sessions & $\begin{array}{l}\text { Reduce phobia in } \\
\text { ASD patients with }\end{array}$ & Target behavior rating & $\begin{array}{l}\text { Rating of specific phobia } \\
\text { change }\end{array}$ & ERS & 1 & 0.39 \\
\hline & & & & & & anxiety disorder & Total fearfulness & FSSC-R & & -0.2 & 0.37 \\
\hline & & & & & & & Intense fears & & & -0.29 & 0.37 \\
\hline & & & & & & & Total anxiety score, parent & SCAS-P & & 0.21 & 0.37 \\
\hline & & & & & & & Total anxiety score, child & SCAS-C & & -0.04 & 0.37 \\
\hline & & & & & & & Formal activity, diversity & CAPE: participation in a & & -0.14 & 0.37 \\
\hline & & & & & & & Formal activity, intensity & range of solitary and group & & -0.1 & 0.37 \\
\hline & & & & & & & Informal activity, diversity & voluntary activities & & -0.24 & 0.37 \\
\hline & & & & & & & Informal activity, intensity & & & -0.28 & 0.37 \\
\hline & & & & & & & & & Overall & 0 & 0.44 \\
\hline \multirow[t]{7}{*}{ Smith et al. (91) } & $24.9(6.7)$ & 16 & 10 & $\begin{array}{l}\text { Being interviewed by } \\
\text { VA in VR-JIT }\end{array}$ & $10 \mathrm{~h}$ & $\begin{array}{l}\text { Improving job } \\
\text { interviewing and }\end{array}$ & $\begin{array}{l}\text { Role-play performance total } \\
\text { score }\end{array}$ & Standardized role-plays & SCS & 0.52 & 0.43 \\
\hline & & & & computer software & & vocational skills & Job interview content score & & & 0.39 & 0.43 \\
\hline & & & & & & & Hard worker & & & 0.58 & 0.43 \\
\hline & & & & & & & Easy to work with/teamwork & & & 0.32 & 0.42 \\
\hline & & & & & & & Sounding professional & & & 0.25 & 0.42 \\
\hline & & & & & & & Negotiation skills & & & 0.32 & 0.42 \\
\hline & & & & & & & $\begin{array}{l}\text { Interviewee performance } \\
\text { score }\end{array}$ & $\begin{array}{l}\text { Training Experience } \\
\text { Questionnaire by } \\
\text { interviewee }\end{array}$ & & 0.49 & 0.43 \\
\hline
\end{tabular}


TABLE 2 | Continued

\begin{tabular}{|c|c|c|c|c|c|c|c|c|c|c|c|}
\hline \multirow[t]{2}{*}{ References } & \multirow{2}{*}{$\frac{\text { Age }}{\text { Mean (SD) }}$} & \multicolumn{2}{|c|}{ Number } & \multirow[t]{2}{*}{ Methodic details } & \multirow{2}{*}{$\begin{array}{l}\text { Application } \\
\text { details }\end{array}$} & \multirow[t]{2}{*}{ Study purpose } & \multicolumn{5}{|c|}{ Outcome measure } \\
\hline & & NI & NC & & & & Name & Details & Skill & $g$ & SEg \\
\hline & & & & & & & Sharing things positively & & & 0.73 & 0.44 \\
\hline & & & & & & & Sounding honest & & & 0 & 0.42 \\
\hline & & & & & & & Sounding interested in job & & & 0.26 & 0.42 \\
\hline & & & & & & & Comfort level & & & 0.46 & 0.43 \\
\hline & & & & & & & Establishing overall rapport & & & 0.35 & 0.42 \\
\hline & & & & & & & $\begin{array}{l}\text { Job interview } \\
\text { self-confidence rating }\end{array}$ & Self-confidence measure & & 0.61 & 0.43 \\
\hline & & & & & & & & & Overall & 0.4 & 0.48 \\
\hline \multirow[t]{4}{*}{ Smith et al. (90) } & \multirow[t]{4}{*}{$25(6.9)$} & \multirow[t]{4}{*}{15} & \multirow[t]{4}{*}{8} & \multirow{4}{*}{$\begin{array}{l}\text { Being interviewed by } \\
\text { VA in VR-JIT } \\
\text { computer software }\end{array}$} & \multirow[t]{4}{*}{$N / S$} & \multirow{4}{*}{$\begin{array}{l}\text { Improving job } \\
\text { interviewing skills }\end{array}$} & Likert score & Self-confidence & SCS & 0.82 & 0.48 \\
\hline & & & & & & & Weeks looking for a job & & & 0.23 & 0.46 \\
\hline & & & & & & & Completed interviews & & & 0.08 & 0.46 \\
\hline & & & & & & & & & Overall & 0.37 & 0.52 \\
\hline \multirow[t]{3}{*}{$\begin{array}{l}\text { Strickland et al. } \\
(97)\end{array}$} & \multirow[t]{3}{*}{$\begin{array}{l}18.21 \\
(1.03)\end{array}$} & \multirow[t]{3}{*}{11} & \multirow[t]{3}{*}{11} & \multirow{3}{*}{$\begin{array}{l}\text { Being interviewed by } \\
\text { VC in JobTIPS } \\
\text { computer program }\end{array}$} & \multirow[t]{3}{*}{ One session } & \multirow[t]{3}{*}{$\begin{array}{l}\text { Enhancing job } \\
\text { finding skills }\end{array}$} & Response content scale & $\begin{array}{l}\text { Content of the participant's } \\
\text { responses }\end{array}$ & DLS & 2.81 & 0.68 \\
\hline & & & & & & & Response delivery scale & $\begin{array}{l}\text { Behaviors related to } \\
\text { greetings and farewells }\end{array}$ & & 0.81 & 0.47 \\
\hline & & & & & & & & & Overall & 1.8 & 0.63 \\
\hline \multirow[t]{3}{*}{ Yuan et al. (84) } & \multirow[t]{3}{*}{$8.97(1.1)$} & \multirow[t]{3}{*}{36} & \multirow[t]{3}{*}{36} & \multirow[t]{3}{*}{$\begin{array}{l}\text { Social scenarios in } \\
\text { four-sided CAVE }\end{array}$} & \multirow[t]{3}{*}{ 1,1-h session } & \multirow{3}{*}{$\begin{array}{l}\text { Enhancing } \\
\text { emotional and } \\
\text { social skills }\end{array}$} & PEP-3 affective expressions & $\begin{array}{l}\text { Emotion expression and } \\
\text { regulation }\end{array}$ & ERS & 0.54 & 0.24 \\
\hline & & & & & & & PEP-3 social reciprocity & $\begin{array}{l}\text { Social interaction and } \\
\text { adaptation }\end{array}$ & SCS & 0.66 & 0.25 \\
\hline & & & & & & & & & Overall & 0.6 & 0.34 \\
\hline \multirow[t]{5}{*}{ Zhang et al. (86) } & \multirow[t]{5}{*}{$4(1.21)$} & \multirow[t]{5}{*}{6} & 5 & Quiver Vision & 20 weeks, two & Enhance social & Social score & ASI disorder score & SCS & 0.14 & 0.69 \\
\hline & & & & $\begin{array}{l}\text { augmented reality } \\
\text { android app }\end{array}$ & $\begin{array}{l}\text { 15-min sessions } \\
\text { per week }\end{array}$ & skills & $\begin{array}{l}\text { Communication and } \\
\text { language }\end{array}$ & & & 0.14 & 0.69 \\
\hline & & & & & & & Anticipation and flexibility & & & -0.28 & 0.69 \\
\hline & & & & & & & Symbolization & & & -0.2 & 0.69 \\
\hline & & & & & & & & & Overall & -0.05 & 0.73 \\
\hline
\end{tabular}

ABAS, Adaptive Behavior Assessment System; CAVE, Cave Automatic Virtual Environment; CS, cognitive skills; DLS, daily living skills; ERS, emotion regulation and recognition skills; FSSC-R, Fear Survey Schedule for Children-Revised; NC, number of participants in control group: NI, number of participants in intervention group; PEP. psychoeducational profile; SCAS C. Spence Children's Anxiety Scale-Child Version; SCAS P. Spence Children's Anxiety Scale-Parent Version; SCS, social and communication skills; TOGGS, Test of Grocery Shopping Skills; VA, virtual avatar; VAP-S, virtual action planning supermarket; VC, virtual character; VR-JIT, Virtual Reality Job Interview Training. The bold values represent overall effectiveness for each study. 


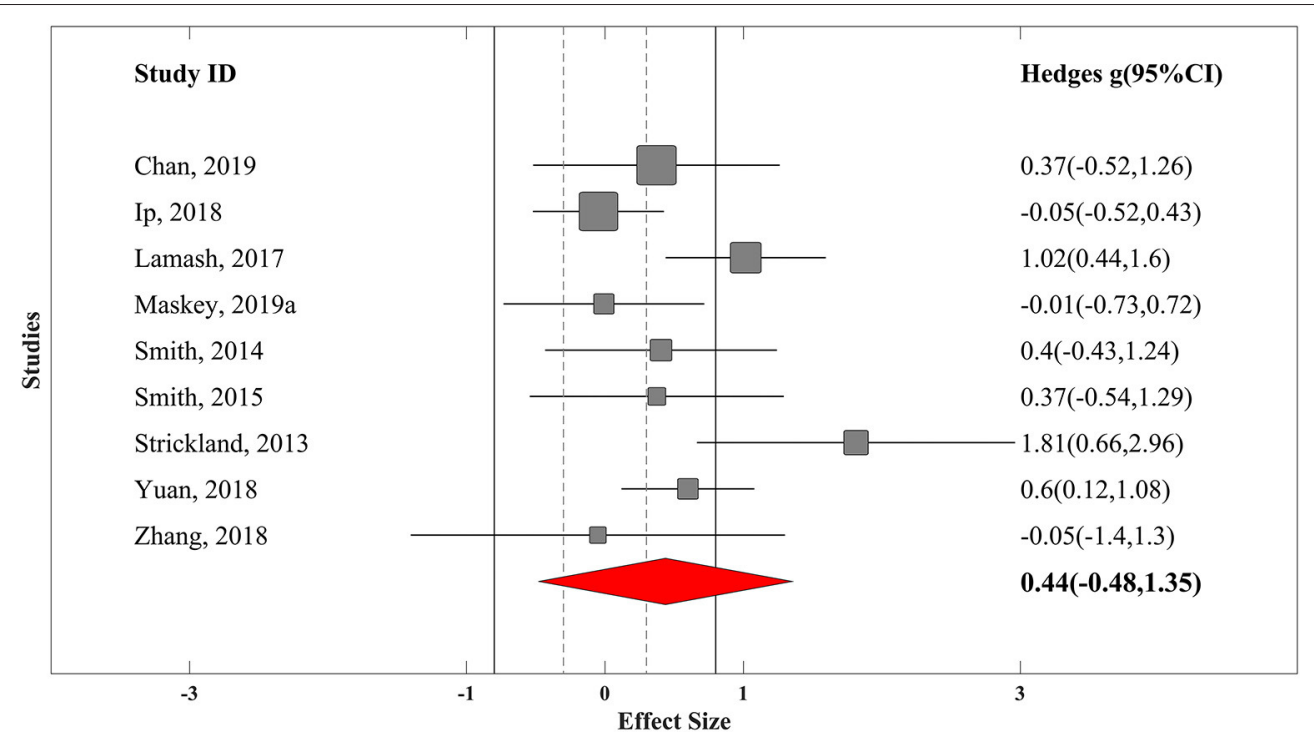

FIGURE 2 | Forest plot of overall effectiveness of VR training for controlled trials with 95\% confidence interval. Solid vertical lines represent strong effect size boundary ( $g=-0.8$ and 0.8), and dashed vertical lines represent weak effect size boundary $(g=-0.3$ and 0.3$)$.

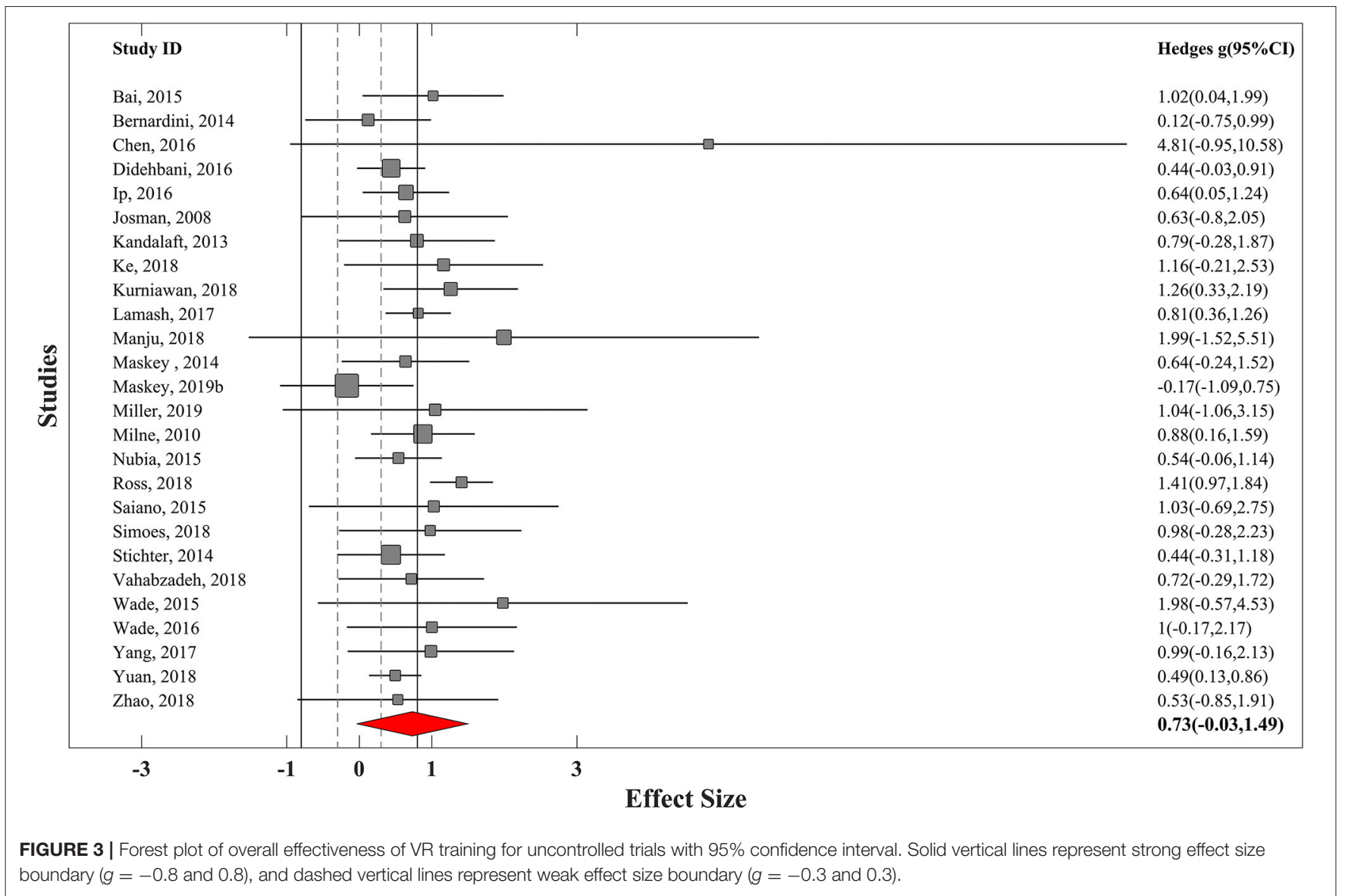

uncontrolled trials unlike substantial decline from unscreened to screened trials in controlled interventions. Because of very small sample size of unscreened controlled trials, the results derived from them seem less reliable, although more cautions should be devoted to screening population before intervention. 


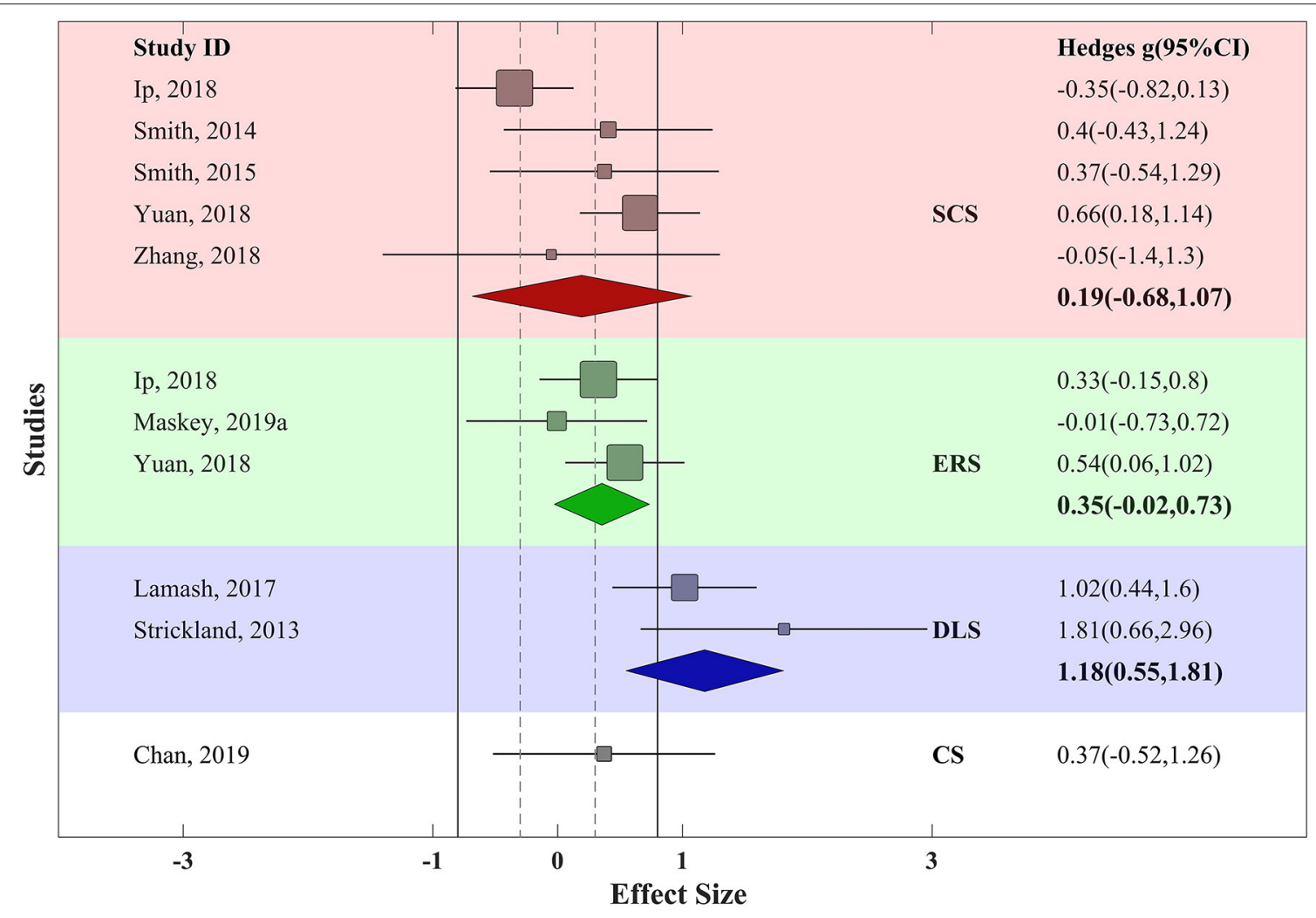

FIGURE 4 | Funnel plot for VR training effectiveness of both uncontrolled and controlled trials with pseudo-95\% confidence interval. Red area represents SCS; green area represents ERS; blue area represents DLS; and yellow area represents CS. Solid vertical lines represent strong effect size boundary ( $g=-0.8$ and 0.8$)$, and dashed vertical lines represent weak effect size boundary $(g=-0.3$ and 0.3). Filled and empty circles represent Hedges $g$ value of uncontrolled and controlled trials, respectively. Solid lines represent $g$ with 95\% confidence interval of uncontrolled trials and dashed lines represent $g$ with 95\% confidence interval of controlled trials.

\section{Subgroup Meta-Analysis}

The results of the subgroup analysis for controlled trials would be underpowered and misleading because of its small sample size, so the conclusions of subgroup meta-analysis and metaregression are limited to the data of uncontrolled trials. By the way, the results of these analyses for controlled trials are available in Supplementary Tables 4, 5.

We performed a subgroup meta-analysis for the categorical moderators described in Methods. Results showed that the overall ESs that had been computed based on the data obtained from non-formal measures were somehow larger than those obtained from formal measures $\left(g=0.93 k=11\right.$, and $\tau^{2}=0.2$ for nonformal and $g=0.66, k=15$, and $\tau^{2}=0.09$ for formal trials). This can be due to the customized measurements that suit the intervention design, less validity of measures, and susceptibility to the rater bias. Regarding the frequency of each measure's application for categories, most of them had applied formal measures except DLS, which application of non-formal measures was more frequent. In DLS, the summary ES was a bit larger for formal measures, although it was derived from only three trials ( $g=1.24, k=4$, and $\tau^{2}=0.55$ for formal and $g=1.06, k$ $=5$, and $\tau^{2}=0.28$ for non-formal measures). For the type of technology (VR or AR), AR interventions led to a larger overall summary $\mathrm{ES}\left(g=0.91, k=5\right.$, and $\tau^{2}=0.3$ for AR and $g=0.71$, $k=21$, and $\tau^{2}=0.1$ for VR). The most of AR interventions were applied for CS that showed more effective training in this skill than $\operatorname{VR}\left(g=0.72, k=3\right.$, and $\tau^{2}=0.15$ for AR and $g=0.33, k$ $=4$, and $\tau^{2}=0.01$ for VR). Regarding intervention effectiveness for age categories, results showed that skill acquiring, in general, got better as the participants got older $\left(g=0.8, k=4\right.$, and $\tau^{2}$ $=0.13$ for ages $4-8$ years; $g=0.57, k=7$, and $\tau^{2}=0.04$ for ages $8-12$ years; $g=0.84, k=7$, and $\tau^{2}=0.09$ for ages $12-$ 16 years; and $g=0.85, k=6$, and $\tau^{2}=0.36$ for ages $>16$ years). Skill categories followed the same trend as the strongest effectiveness observed in the age older than 16 years for all of them $\left(g=0.98, k=2\right.$, and $\tau^{2}=0.3$ for SCS; $g=0.46, k=$ 4 , and $\tau^{2}=0.03$ for ERS; and $g=1.33, k=3$, and $\tau^{2}=0.82$ for DLS for other age groups; Table 3 ). It is also noteworthy to say that effectiveness was relatively strong for CS in participants aged 4-8 years, which was the major outcome addressed by our included trials in this age group $\left(g=0.77, k=3, \tau^{2}=0.15\right)$. These results point to a more favorable effect of VR interventions for older patients. Subgroup analysis for comorbidity revealed considerable decline in training effectiveness on ASD patients with concomitant comorbidity as $g=0.77$ in 20 trials with $\tau^{2}$ $=0.13$ in which patients did not have any specified comorbidity dropped to $g=0.6$ in six trials with $\tau^{2}=0.03$ in which patients had some type of comorbidity alongside their main disease. This 


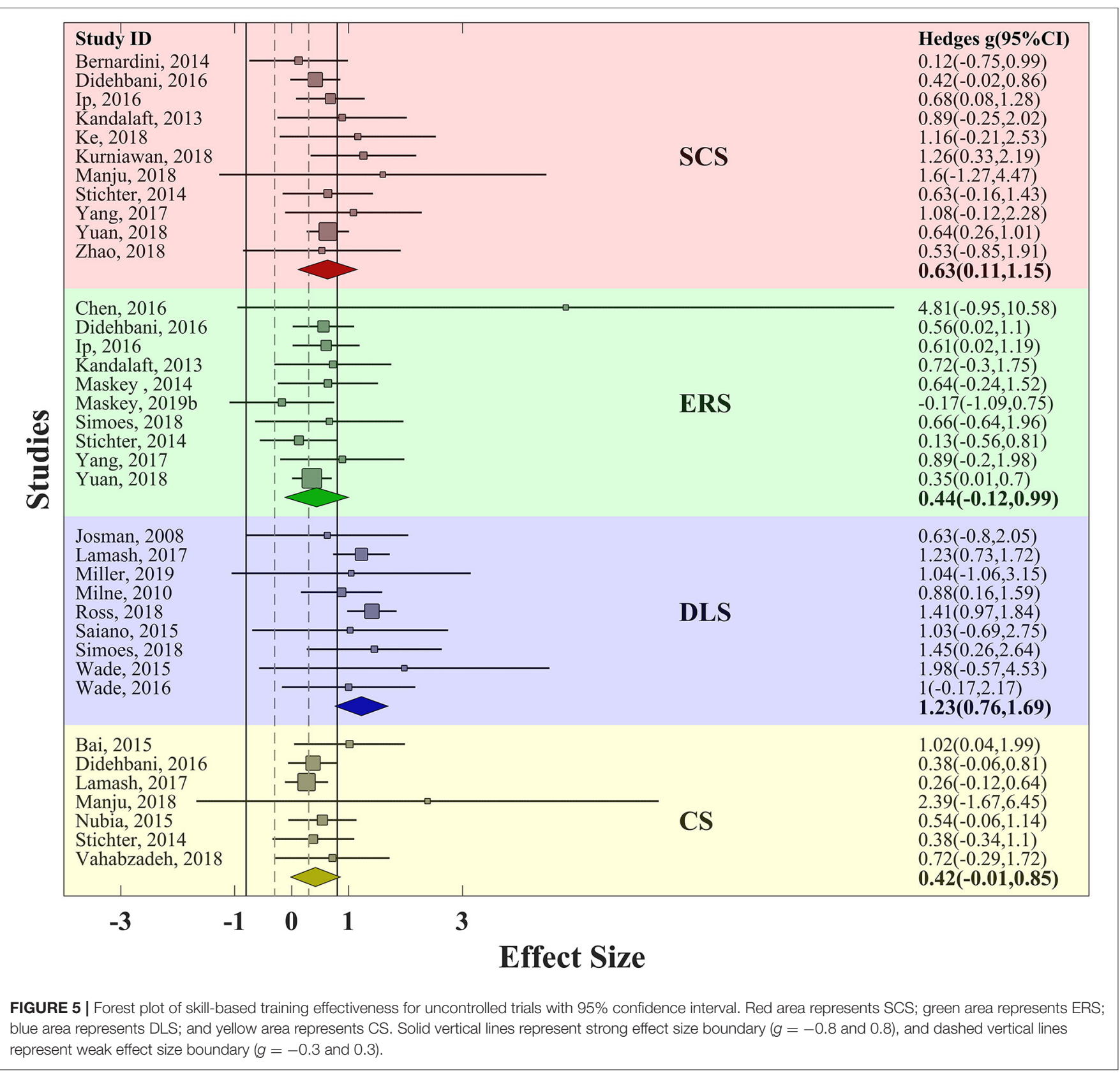

effect was even more sophisticated in controlled trials so that $g=$ 0.57 in six trials with $\tau^{2}=0.08$ without specified comorbidity reduced to $g=0.11$ in three trials with $\tau^{2}=0.03$ in which a comorbidity was diagnosed. The full Results can be seen in Table 3.

\section{Meta-Regression}

To see if there is any significant interaction between continuous moderators (number of sessions, sex, and publication date) and effectiveness of the intervention, we opted for univariate linear regression on weighted ESs as a function of each of these moderators for all designs and skill categories (Table 4). Significant relationship was found in publication date for overall
$(\mathrm{N}=122$, betal $=0.4, p=0.02)$ and DLS $(\mathrm{N}=30$, beta1 $=0.95, p=0.006$ ), which show that over time, intervention qualities have been likely to be improved as the technology has been advancing; there was no significant association between the number of sessions or gender and computed ESs in any of outcome categories.

\section{Publication Bias}

Visual inspection of the funnel plot (Figure 6) for both controlled and uncontrolled overall ESs pointed to a symmetrical funnel for both trials. To validate this conclusion statistically, we applied Egger regression intercept test. The test results corroborated the visual inspection by revealing no significant bias for uncontrolled 
TABLE 3 | Subgroup meta-analysis results for type of measure, type of technology, and age moderators.

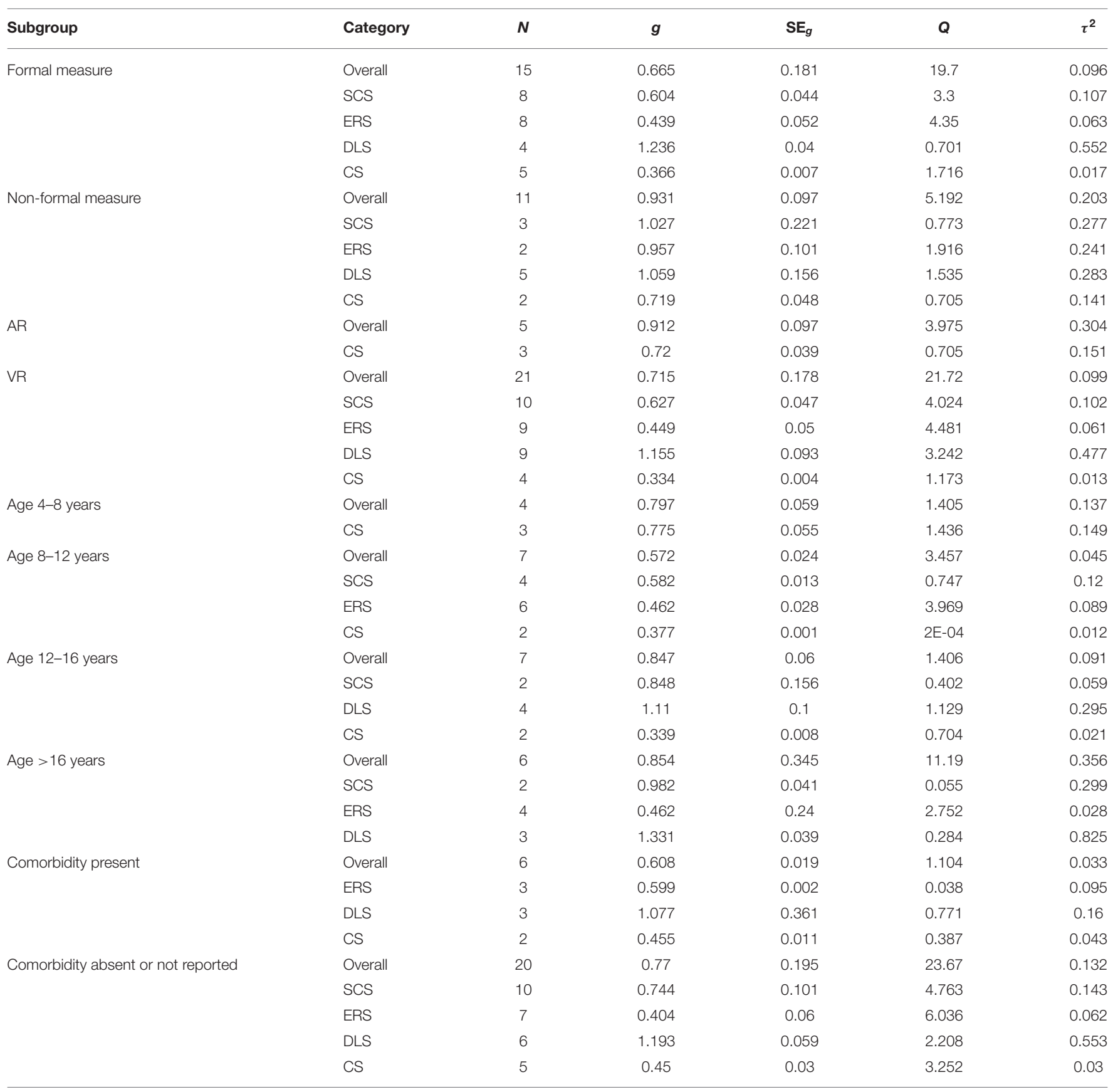

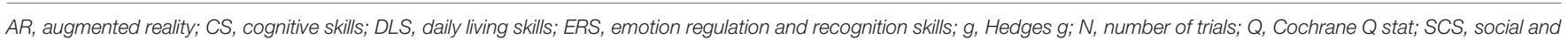
communication skills; $S E_{g}$, standard error of $g$; VR, virtual reality.

and controlled trials [intercept $=0.27(p=0.24)$ for uncontrolled and intercept $=0.1(p=0.88)$ for controlled trials]. This implies that drawn conclusions are robust and reliable.

Comparing effectiveness of VR training with some of conventional behavioral programs that were addressed by three meta-analysis studies, we observed a comparable moderate effectiveness of our study with the most of clinical targets appraised by them. One exception was effectiveness of early intensive behavioral intervention on full-scale IQ of patients with ASD, which was proven to be strong ( $g=1.1$ of nine controlled studies). The other interesting finding was that TEACCH and ABA programs were not effective in improving daily living skills ( $g=0.34$ from 6 and $g=0.14$ from 29 studies, respectively), while the effectiveness of VR training on this clinical target was very strong as it was observed in both controlled and uncontrolled trials. For full results on this part, see Table 5. 
TABLE 4 | Metaregression results for number of sessions, sex, and publication date moderators.

\begin{tabular}{|c|c|c|c|c|}
\hline Moderator & Skill & $n$ & Slope & $p$ \\
\hline \multirow[t]{5}{*}{ No. of sessions } & Overall & 122 & -0.035 & 0.4912 \\
\hline & SCS & 38 & 0.0086 & 0.8766 \\
\hline & ERS & 27 & 0.0297 & 0.6552 \\
\hline & DLS & 30 & 0.5425 & 0.3548 \\
\hline & CS & 27 & -0.148 & 0.188 \\
\hline \multirow[t]{5}{*}{ Gender } & Overall & 122 & 4.5146 & 0.2318 \\
\hline & SCS & 38 & -2.162 & 0.8148 \\
\hline & ERS & 27 & 6.6054 & 0.0935 \\
\hline & DLS & 30 & -0.25 & 0.9892 \\
\hline & CS & 27 & -18.7 & 0.1484 \\
\hline \multirow[t]{5}{*}{ Publication date } & Overall* $^{*}$ & 122 & 0.4021 & $0.0219^{*}$ \\
\hline & SCS & 38 & 0.212 & 0.5024 \\
\hline & ERS & 27 & -0.237 & 0.4484 \\
\hline & $\mathrm{DLS}^{\star}$ & 30 & 0.9515 & $0.0067^{\star}$ \\
\hline & CS & 27 & 0.7987 & 0.2738 \\
\hline
\end{tabular}

*Significant values with $p<0.05$.

CS, cognitive skills; DLS, daily living skills; ERS, emotion regulation and recognition skills;

$N$, number of outcomes in each group; SCS, social and communication skills.

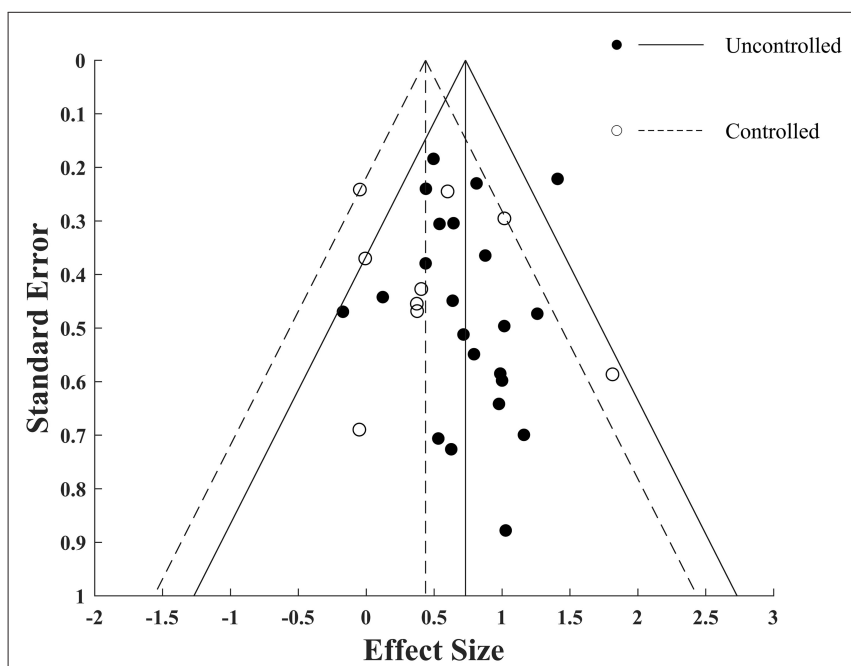

FIGURE 6 | Funnel plot for VR training effectiveness of both uncontrolled and controlled trials with pseudo 95\% confidence interval. Filled and empty circles represent Hedges $g$ value of uncontrolled and controlled trials respectively. Solid lines represent $g$ with $95 \%$ confidence interval of uncontrolled trials and dashed lines represent $g$ with 95\% confidence interval of controlled trials.

\section{DISCUSSION}

We performed a systematic review and meta-analysis on the effectiveness of applying VR-based therapeutic interventions on the alleviation of deficits in ASD patients. Based on the results of 26 uncontrolled and nine controlled trials, we concluded that VR technology can be a viable tool for designing interventions aimed at enhancing and improving different skills in people suffering from ASD at any age. To our knowledge, this is the first meta-analysis focusing exclusively on the effectiveness of VR-based interventions for training ASD patients. Although there are some meta-analysis studies available on the same topic with various types of new technologies that may (46, 63) or may not (103-105) include VR, the number of trials that methodologically focused on VR was not large enough to draw rigorous conclusions around their efficacy. The increase in the number of VR interventions has been conducted recently; besides, its public availability has justified the need for this study. Overall moderate effectiveness of VR interventions that we observed in this study is in line with the results of previously mentioned studies. Our study shows moderate effectiveness ( $g=$ 0.44) of VR interventions based on controlled trials and strong effectiveness ( $g=0.73$ ) based on uncontrolled trials. Although the number of uncontrolled trials was conspicuously larger than controlled ones (26-9), a more credible design of controlled trials leads us to the point to claim moderate effectiveness of VR-based training in individuals with ASD.

Low heterogeneity in uncontrolled trials would provide further support for the conclusion drawn from these trials. Moderate heterogeneity in controlled trials cast doubts on interpretation of their results that could be explained by their relative small sample size (only nine trials comparing to 26 uncontrolled trials) and also the heterogeneity of control groups.

Further analysis of entailed categories of skills revealed relatively the same moderate effectiveness of intervention for SCS, ERS, and CS except daily living skills that outperformed other categories with promising large effectiveness in both design groups. This effect was proven to be consistent across different trials with different designs as heterogeneity was low for both of them. This finding can be specifically of interest because of the more reflective nature of DL skills, which means that they are gained and generalized in later stages of cognitive development, and ASD subjects are required to be trained for it similarly to their neurotypical counterparts. Unlike reflective skills, communication or emotional skills are more intuitive in the sense that they are generally gained in the early stages of development without any specific effort. It is also possible that the observed large effect originated from the larger mean age of participants in this outcome category comparing to other categories, which have given them superiority and dexterity in learning and practicing skills. Nevertheless, significantly larger effectiveness of VR training for DLS compared to others is persuasive enough for us to put forward the hypothesis that reflective skills hold more potential toward improvement by training than intuitive skills. Here we may have corroborated this hypothesis for VR-based training. On the other hand, medium effectiveness for communication, emotion, and CS may be due to the complex mental nature of these skills. As it is reflected in the recent systematic review (62), although these skills have been the center of attention in most studies, just partial improvements have been made. Knowing this, in future efforts, a more elaborated intervention design seems necessary to ascertain the effectiveness of VR training on these types of skills. Taken together, we encourage psychiatrists and educators of people with ASD to practice this type of technology with more focus on daily living skills. 
TABLE 5 | Characteristics and effectiveness of three of the conventional rehabilitation programs.

\begin{tabular}{|c|c|c|c|c|c|c|c|c|}
\hline References & Intervention & $\begin{array}{c}\text { Publication } \\
\text { year }\end{array}$ & $\begin{array}{l}\text { No. of } \\
\text { studies }\end{array}$ & $\begin{array}{c}\text { Case } \\
\text { number }\end{array}$ & Study design & Outcome measure & $\begin{array}{l}\text { ES } \\
\text { statistic }\end{array}$ & ES \\
\hline \multirow{12}{*}{$\begin{array}{l}\text { Virues-Ortega } \\
\text { et al. (3) }\end{array}$} & \multirow[t]{12}{*}{ TEACCH } & \multirow[t]{12}{*}{2013} & 13 & 172 & \multirow[t]{12}{*}{ Uncontrolled } & Overall & \multirow[t]{12}{*}{ Cohen $d$} & 0.47 \\
\hline & & & \multirow[t]{5}{*}{6} & \multirow[t]{5}{*}{93} & & Eye-hand coordination & & 0.26 \\
\hline & & & & & & Motor functioning & & 0.36 \\
\hline & & & & & & Gross motor function & & 0.58 \\
\hline & & & & & & Imitation & & 0.41 \\
\hline & & & & & & Perception & & 0.4 \\
\hline & & & 5 & 74 & & Communication skills & & 0.34 \\
\hline & & & 6 & 81 & & Daily living skills & & 0.32 \\
\hline & & & 5 & 74 & & Social functioning & & 0.64 \\
\hline & & & 5 & 43 & & Cognitive functioning & & 0.41 \\
\hline & & & 9 & 121 & & Verbal skills & & 0.36 \\
\hline & & & 4 & 44 & & Maladaptive behaviors & & -0.92 \\
\hline \multirow[t]{2}{*}{ Eldevik et al. (4) } & \multirow{2}{*}{$\begin{array}{l}\text { Early Intensive } \\
\text { Behavioral }\end{array}$} & \multirow[t]{2}{*}{2013} & \multirow[t]{2}{*}{9} & \multirow{2}{*}{$\begin{array}{l}153 / 105 \\
\text { (control) }\end{array}$} & \multirow[t]{2}{*}{ Controlled } & Full-scale IQ & \multirow[t]{2}{*}{ Hedges $g$} & 1.1 \\
\hline & & & & & & Adaptive behavior & & 0.66 \\
\hline \multirow{8}{*}{$\begin{array}{l}\text { Makrygianni } \\
\text { et al. (5) }\end{array}$} & \multirow{8}{*}{$\begin{array}{l}\text { Applied Behavior } \\
\text { Analytic interventions }\end{array}$} & \multirow[t]{8}{*}{2018} & \multirow[t]{8}{*}{29} & \multirow[t]{8}{*}{831} & \multirow[t]{8}{*}{ Uncontrolled } & Intellectual abilities & \multirow[t]{8}{*}{ Hedges $g$} & 0.74 \\
\hline & & & & & & Communication skills & & 0.65 \\
\hline & & & & & & Expressive-language skills & & 0.742 \\
\hline & & & & & & Receptive-language skills & & 0.597 \\
\hline & & & & & & Non-verbal IQ & & 0.463 \\
\hline & & & & & & Adaptive behavior & & 0.422 \\
\hline & & & & & & Socialization & & 0.444 \\
\hline & & & & & & Daily living skills & & 0.138 \\
\hline
\end{tabular}

ES, effect size estimate; TEACCH, Treatment and Education of Autistic and Related Communication Handicapped Children.

The results of our subgroup analysis are merely discussed on overall outcomes of uncontrolled trials. The small sample size of other subgroups precluded us from drawing a strong conclusion for them. The effectiveness of an intervention based on formal and non-formal measures was roughly similar and around moderate range, which indicates that our results might not be affected with the existence of non-formal assessments. Apart from that, cautious interpretation of informal measures should be considered, and it is possible that defined informal measures could be biased. The effectiveness of AR was similar and even a little larger than VR interventions. Although the sample size of the AR subgroup was relatively small, considering its low heterogeneity, the resultant conclusion on this subgroup can be reliable. This is particularly important because AR interventions can be conducted by means of AR-enabled mobile phones, which is ubiquitous nowadays providing more controlled interventions for larger populations of patients with ASD. The superiority of AR can be assigned to its simplicity in design and convenience of use compared to VR in which tasks are designed and applied in more complex environments with more parameters to understand and deal with. This simplicity can lead to a sooner and better engagement of participants in the task specifically for younger children.

The results of the subgroup analysis for age categories revealed that performance improves as the age gets larger. Particularly, it is important to note that this improvement is happening not only in daily living skills, which are reflective skills and later in development, but also in other intuitive areas, such as social skills and emotion recognition skills. This phenomenon may be induced by two factors. First, patients with autism presumably develop a kind of mechanism to overcome the deficits primarily caused by ASD, and so they assimilate to their milieu as they age. Second, older patients may have the advantage that they understand the task and VR environment better, and so they interact with it more efficiently, resulting in improved performance. In the first age category (4-8 years old), a notable relatively strong effectiveness was observed in CS, which was the only addressed area in this age group. Despite the small sample size, the relatively large ES was persuasive enough for us to consider it. This large effect may also be seen in other areas of SCS, ERS, and DLS; therefore, we encourage the scientific community to target their interventions on these areas too.

We observed a substantial decline in the effectiveness of training on patients who suffered from some sort of concomitant comorbidity along with ASD. This phenomenon was particularly interesting in controlled trials as observed moderate effectiveness of training on ASD patients without other comorbidity was completely vanished when concomitant comorbidity was taken into account. This alarms the future practitioners who are trying to improve skills in patients with ASD by means of VR interventions to carefully screen their target patients for having other concomitant comorbidity.

The effectiveness of training for HFASD patients was moderate to large, which was equivalent to its overall value 
regardless of the level of the disorder. In most of the studies, whether composed of a combination of LFASD and HFASD participants or the level of disease was not specified, direct association between level of disease and effectiveness of intervention could not be derived. For this reason, we call for papers with more focus on defining the level and functionality of disease in included participants for better characterizing the target population of intervention.

The results of meta-regression revealed a significant correlation between publication date and VR training effectiveness, which can be interpreted under improvement in the design and conduction of VR interventions over time. Surprisingly, effectiveness was not influenced by the total number of intervention sessions. It is important to mind that the session's duration and its distribution over the course of intervention were unreported or highly heterogeneous among the trials, and therefore, the net number of sessions might not be a good representative for intensity and quality of intervention. For this reason, hesitant interpretations warrant caution, and more controlled interventions in terms of design, duration, and longevity are needed for more conclusive interpretations on this matter. The sex of participants was not a significant moderator of the results in our study as it is not seen in other studies of this kind.

Comparing the results of the current meta-analysis with those of more conventional training programs (Table 5), it is evident that VR-based training is at least as effective in most study endpoints as traditional programs. In addition, the more flexible and favorable nature of VR leads to more elaborate task designs, more enthusiasm in participants to do those tasks, and ultimately more accurate assessments of improvement. These factors together might result in more ecological validity of VR-based experiments and more reliability of their results.

The strong effectiveness of daily living skills (reflected in both controlled and uncontrolled trials) was achieved only through VR-based training, not conventional training. It is therefore sensible to use VR to design rehabilitation programs aimed at daily living skills in clinical practice. In the other clinical targets, a further improvement in the design and application of VR technology is still required.

\section{Limitations and Recommendations for Future Research}

Most of our included studies were uncontrolled pretest-posttest trials. It has been argued that these types of trials should be avoided in meta-analysis as the pretest and posttest scores are not independent of each other, and thus, accurate calculation of SMDs requires knowledge of correlation value between these two scores, which is not provided in most of the studies (106). Aside from that, perhaps due to differing epistemological bases of research being carried out in this broad domain, most of the studies have adopted this type of design for their intervention, which makes considering this massive body of data for analysis inevitable. Here, we have done all the calculations with the premise of independent pretest and posttest scores (zero correlation), which is subsequently leading to the largest pooled variance and thus the smallest possible value of computed ES.
For this reason, we claim that our applied method is the most parsimonious one avoiding any overestimation in computing the ESs.

Although the number of participants in most studies was rather low, and so their estimations would not be adequately powered, its effect might be compensated by a considerably large number of included trials. Many trials had not screened participants for critical contributing factors that could affect the outcome. This issue seemed to be a challenge for our results. However, later analysis relieved this by showing that the summary ES of those trials that screened included population did not deviate drastically from those who did not perform this screening.

The type of VR technology applied by studies was diverse enough to prevent us from establishing a systematic relationship between the technology type and its effectiveness, so further studies are required to investigate such a connection. To our surprise, restricted and repetitive behavior, which is one of the core symptoms of ASD, was not addressed by any of studies, so more experiments are encouraged to be targeted in this area in the future works. Follow-up assessment of participants was performed in an only limited number of trials; therefore, the maintenance of treatment effects, although important, could not be assessed in our study.

\section{CONCLUSIONS}

The current findings support the effectiveness of VR training to improve ASD-related disabilities. The strong observed effectiveness for daily living skills could justify the application of VR interventions in clinical practice. For future research, the designed experiments need to be more controlled in terms of selection of participants, type and duration of intervention, and choice of a measurement tool, and finally, more efforts should be devoted to follow-up assessments carried out weeks or months after the end of the intervention to ensure that the effects of training are consolidated and maintained.

\section{DATA AVAILABILITY STATEMENT}

The original contributions presented in the study are included in the article/Supplementary Material, further inquiries can be directed to the corresponding author/s.

\section{AUTHOR CONTRIBUTIONS}

A-HV and FA designed the study and wrote the protocol. BK, RK, and MR conducted literature searches and provided summaries of previous research studies. BK analyzed data and produced figures. BK and RK wrote the first draft of the manuscript. A-HV supervised the study. All authors contributed to and have approved the final manuscript.

\section{SUPPLEMENTARY MATERIAL}

The Supplementary Material for this article can be found online at: https://www.frontiersin.org/articles/10.3389/fpsyt. 2021.665326/full\#supplementary-material 


\section{REFERENCES}

1. American Psychiatric Association. Diagnostic and Statistical Manual of Mental Disorders (DSM- $\left.5^{\circledR}\right)$. Washington DC: American Psychiatric Pub. (2013). doi: 10.1176/appi.books.9780890425596

2. Baio J. Prevalence of autism spectrum disorder among children aged 8 years - autism and developmental disabilities monitoring network, 11 sites, United States, 2010. Autism and Developmental Disabilities Monitoring Network. Surveillance Year 2010 Principal Investigators. In: Centers for Disease Control and Prevention (U.S.), National Center on Birth Defects and Developmental Disabilities (Centers for Disease Control and Prevention), editors, MMWR Surveillance Summaries: Morbidity and Mortality Weekly Report Surveillance Summaries. (2014). p. 63. Available online at: https:// stacks.cdc.gov/view/cdc/22182 (accessed May 31, 2021).

3. Virues-Ortega J, Julio FM, Pastor-Barriuso R. The TEACCH program for children and adults with autism: a meta-analysis of intervention studies. Clin Psychol Rev. (2013) 33:940-53. doi: 10.1016/j.cpr.2013.07.005

4. Eldevik S, Hastings RP, Hughes JC, Jahr E, Eikeseth S, Cross S. Metaanalysis of early intensive behavioral intervention for children with autism. J Clin Child Adolesc Psychol. (2009) 38:439-50. doi: 10.1080/153744109028 51739

5. Makrygianni MK, Gena A, Katoudi S, Galanis P. The effectiveness of applied behavior analytic interventions for children with Autism Spectrum Disorder: a meta-analytic study. Res Autism Spectr Disord. (2018) 51:18-31. doi: 10.1016/j.rasd.2018.03.006

6. Dawson G, Rogers S, Munson J, Smith M, Winter J, Greenson J, et al. Randomized, controlled trial of an intervention for toddlers with autism: the Early Start Denver Model. Pediatrics. (2010) 125:e17-23. doi: 10.1542/peds.2009-0958

7. Weitlauf AS, McPheeters ML, Peters B, Sathe N, Travis R, Aiello R, et al. Therapies for Children With Autism Spectrum Disorder: Behavioral Interventions Update. Rockville, MD: Agency for Healthcare Research and Quality (2014). Available online at: http://www.ncbi.nlm.nih.gov/books/ NBK241444/ (accessed April 22, 2021).

8. Miller HL, Bugnariu NL. Level of immersion in virtual environments impacts the ability to assess and teach social skills in autism spectrum disorder. Cyberpsychol Behav Soc Netw. (2016) 19:246-56. doi: $10.1089 /$ cyber.2014.0682

9. Billard A, Robins B, Nadel J, Dautenhahn K. Building Robota, a minihumanoid robot for the rehabilitation of children with autism. Assist Technol. (2007) 19:37-49. doi: 10.1080/10400435.2007.10131864

10. Dautenhahn K. Roles and functions of robots in human society: implications from research in autism therapy. Robotica. (2003) 21:443-52. doi: $10.1017 /$ S0263574703004922

11. Moghadas M, Moradi H. Analyzing human-robot interaction using machine vision for autism screening. In: 2018 6th RSI International Conference on Robotics and Mechatronics (IcRoM). Tehran (2018). p. 572-6. doi: 10.1109/ICRoM.2018.8657569

12. Aldi C, Crigler A, Kates-McElrath K, Long B, Smith H, Rehak K, et al. Examining the effects of video modeling and prompts to teach activities of daily living skills. Behav Anal Practice. (2016) 9:384-8. doi: 10.1007/s40617-016-0127-y

13. Golan O, Ashwin E, Granader Y, McClintock S, Day K, Leggett $\mathrm{V}$, et al. Enhancing emotion recognition in children with autism spectrum conditions: an intervention using animated vehicles with real emotional faces. J Autism Dev Disord. (2010) 40:269-79. doi: 10.1007/s10803-009-0862-9

14. Macpherson K, Charlop $\mathrm{MH}$, Miltenberger CA. Using portable video modeling technology to increase the compliment behaviors of children with autism during athletic group play. J Autism Dev Disord. (2015) 45:3836-45. doi: $10.1007 /$ s10803-014-2072-3

15. Burckley E, Tincani M, Guld Fisher A. An iPad ${ }^{\mathrm{TM}}$-based picture and video activity schedule increases community shopping skills of a young adult with autism spectrum disorder and intellectual disability. Dev Neurorehabil. (2015) 18:131-6. doi: 10.3109/17518423.2014.945045

16. Cihak DF, Wright R, Ayres KM. Use of self-modeling static-picture prompts via a handheld computer to facilitate self-monitoring in the general education classroom. Educ Train Autism Dev Disabil. (2010) 45:136-49.

17. El Zein F, Gevarter C, Bryant B, Son S-H, Bryant D, Kim M, et al. A comparison between iPad-Assisted and teacher-directed reading instruction for students with Autism Spectrum Disorder (ASD). J Dev Phys Disabil. (2016) 28:195-215. doi: 10.1007/s10882-015-9458-9

18. Kinsella BG, Chow S, Kushki A. Evaluating the usability of a wearable social skills training technology for children with autism spectrum disorder. Front Robot AI. (2017) 4:31. doi: 10.3389/frobt.2017.00031

19. Parsons S, Cobb S. State-of-the-art of virtual reality technologies for children on the autism spectrum. Eur J Special Needs Educ. (2011) 26:355-66. doi: $10.1080 / 08856257.2011 .593831$

20. Parsons S, Mitchell P. The potential of virtual reality in social skills training for people with autistic spectrum disorders. J Intellect Disabil Res. (2002) 46:430-43. doi: 10.1046/j.1365-2788.2002.00425.x

21. Moore M, Calvert S. Brief report: vocabulary acquisition for children with autism: teacher or computer instruction. J Autism Dev Disord. (2000) 30:35962. doi: 10.1023/A:1005535602064

22. Bernard-Opitz V, Sriram N, Nakhoda-Sapuan S. Enhancing social problem solving in children with autism and normal children through computer-assisted instruction. J Autism Dev Disord. (2001) 31:377-84. doi: 10.1023/A:1010660502130

23. Williams C, Wright B, Callaghan G, Coughlan B. Do children with autism learn to read more readily by computer assisted instruction or traditional book methods? a pilot study. Autism. (2002) 6:71-91. doi: $10.1177 / 1362361302006001006$

24. Cheryl G. Trepagnier. Virtual environments for the investigation and rehabilitation of cognitive and perceptual impairments. NeuroRehabilitation. (1999) 12:63-72. doi: 10.3233/NRE-1999-12107

25. Bellani M, Fornasari L, Chittaro L, Brambilla P. Virtual reality in autism: state of the art. Epidemiol Psychiatr Sci. (2011) 20:235-8. doi: $10.1017 /$ S2045796011000448

26. Georgescu AL, Kuzmanovic B, Roth D, Bente G, Vogeley K. The use of virtual characters to assess and train non-verbal communication in high-functioning autism. Front Hum Neurosci. (2014) 8:807. doi: $10.3389 /$ fnhum.2014.00807

27. Rajendran G. Virtual environments and autism: a developmental psychopathological approach. J Comput Assist Learn. (2013) 29:334-47. doi: $10.1111 /$ jcal.12006

28. Parsons S. Authenticity in virtual reality for assessment and intervention in autism: a conceptual review. Educ Res Rev. (2016) 19:138-57. doi: 10.1016/j.edurev.2016.08.001

29. Kurniawan I. The improvement of autism spectrum disorders on children communication ability with PECS method Multimedia Augmented RealityBased. J Phys. (2018) 2018:12009. doi: 10.1088/1742-6596/947/1/012009

30. Manju T, Padmavathi S, Tamilselvi D. A rehabilitation therapy for autism spectrum disorder using virtual reality. In: Venkataramani GP, Sankaranarayanan K, Mukherjee S, Arputharaj K, Sankara Narayanan S, editors, Smart Secure Systems - IoT and Analytics Perspective. Singapore: Springer Singapore. (2018). p. 328-36. doi: 10.1007/978-981-10-7635-0_26

31. Zhao H, Swanson AR, Weitlauf AS, Warren ZE, Sarkar N. Hand-inhand: a communication-enhancement collaborative virtual reality system for promoting social interaction in children with autism spectrum disorders. IEEE Trans Human-Machine Syst. (2018) 48:136-48. doi: 10.1109/THMS.2018.2791562

32. Bekele E, Crittendon J, Zheng Z, Swanson A, Weitlauf A, Warren Z, et al. Assessing the utility of a virtual environment for enhancing facial affect recognition in adolescents with autism. J Autism Dev Disord. (2014) 44:164150. doi: $10.1007 /$ s10803-014-2035-8

33. Ip HHS, Wong SWL, Chan DFY, Byrne J, Li C, Yuan VSN, et al. Enhance emotional and social adaptation skills for children with autism spectrum disorder: a virtual reality enabled approach. Comput Educ. (2018) 117:1-15. doi: 10.1016/j.compedu.2017.09.010

34. Lorenzo G, Lledo A, Pomares J, Roig R. Design and application of an immersive virtual reality system to enhance emotional skills for children with autism spectrum disorders. Comput Educ. (2016) 98:192-205. doi: 10.1016/j.compedu.2016.03.018 
35. Adjorlu A, Høeg ER, Mangano L, Serafin S. Daily living skills training in virtual reality to help children with autism spectrum disorder in a real shopping scenario. In: 2017 IEEE International Symposium on Mixed and Augmented Reality (ISMAR-Adjunct). Nantes (2017). p. 294-302. doi: 10.1109/ISMAR-Adjunct.2017.93

36. Lamash L, Klinger E, Josman N. Using a virtual supermarket to promote independent functioning among adolescents with Autism Spectrum Disorder. In: 2017 International Conference on Virtual Rehabilitation (ICVR). Montreal (2017). p. 1-7. doi: 10.1109/ICVR.2017.8007467

37. Ross V, Cox DJ, Reeve R, Brown T, Moncrief M, Schmitt R, et al. Measuring the attitudes of novice drivers with autism spectrum disorder as an indication of apprehensive driving: going beyond basic abilities. Autism Int J Res Practice. (2018) 22:62-9. doi: 10.1177/1362361317735959

38. Wade J, Bian D, Fan J, Zhang L, Swanson A, Sarkar M, et al. A virtual reality driving environment for training safe gaze patterns: application in individuals with ASD. In: Antona M, Stephanidis C, editors, Universal Access in Human-Computer Interaction: Access to Learning, Health and Well-Being, UAHCI 2015, PT III. (2015). p. 689-97. (Lecture Notes in Computer Science; vol. 9177). doi: 10.1007/978-3-319-20684-4_66

39. Wade J, Zhang L, Bian D, Fan J, Swanson A, Weitlauf A, et al. A gazecontingent adaptive virtual reality driving environment for intervention in individuals with autism spectrum disorders. ACM Trans Interactive Intelligent Systems. (2016) 6:3. doi: 10.1145/2892636

40. Josman N, Ben-Chaim HM, Friedrich S, Weiss PL. Effectiveness of virtual reality for teaching street-crossing skills to children and adolescents with autism. Int J Disabil Hum Dev. (2008) 7:49-56. doi: 10.1515/IJDHD.2008.7.1.49

41. Saiano M, Pellegrino L, Casadio M, Summa S, Garbarino E, Rossi V, et al. Natural interfaces and virtual environments for the acquisition of street crossing and path following skills in adults with Autism Spectrum Disorders: a feasibility study. J Neuroeng Rehabil. (2015) 12:17. doi: 10.1186/s12984-015-0010-Z

42. Didehbani N, Allen T, Kandalaft M, Krawczyk D, Chapman S. Virtual reality social cognition training for children with high functioning autism. Comput Hum Behav. (2016) 62:703-11. doi: 10.1016/j.chb.2016.04.033

43. Nubia RM, Fabián GR, Wilson RA, Wilmer PB. Development of a mobile application in augmented reality to improve the communication field of autistic children at a Neurorehabilitar Clinic. In: 2015 Workshop on Engineering Applications - International Congress on Engineering (WEA). Bogota (2015). p. 1-6. doi: 10.1109/WEA.2015.7370154

44. Vahabzadeh A, Keshav NU, Salisbury JP, Sahin NT. Improvement of attention-deficit/hyperactivity disorder symptoms in school-aged children, adolescents, and young adults with autism via a digital smartglasses-based socioemotional coaching aid: short-term, uncontrolled pilot study. JMIR Mental Health. (2018) 5:9631. doi: 10.2196/mental.9631

45. Burdea GC, Coiffet P. Virtual Reality Technology. Cambridge, MA: John Wiley \& Sons (2003). doi: 10.1162/105474603322955950

46. Self T, Scudder RR, Weheba G, Crumrine D. A virtual approach to teaching safety skills to children with autism spectrum disorder. Top Lang Disord. (2007) 27:242. doi: 10.1097/01.TLD.0000285358.33545.79

47. Mishkind MC, Norr AM, Katz AC, Reger GM. Review of virtual reality treatment in psychiatry: evidence vs. current diffusion and use. Curr Psychiatry Rep. (2017) 19:80. doi: 10.1007/s11920-017-0836-0

48. Raaja NR, Shiva GS, Mithun P, Vijayabhas PVM. A review on: augmented reality technologies, systems and applications. J Appl Sci. (2014) 14:1485-95. doi: 10.3923/jas.2014.1485.1495

49. Holden MK. Virtual environments for motor rehabilitation: review. CyberPsychol Behav. (2005) 8:187-211. doi: 10.1089/cpb.2005.8.187

50. Weiss PL, Kizony R, Feintuch U, Katz N. Virtual reality in neurorehabilitation. Textbook Neural Repair Rehabil. (2006) 51:182-97. doi: 10.1017/CBO9780511545078.015

51. Iruthayarajah J, McIntyre A, Cotoi A, Macaluso S, Teasell R. The use of virtual reality for balance among individuals with chronic stroke: a systematic review and meta-analysis. Top Stroke Rehabil. (2017) 24:68-79. doi: 10.1080/10749357.2016.1192361

52. Li Z, Han X-G, Sheng J, Ma S-J. Virtual reality for improving balance in patients after stroke: a systematic review and meta-analysis. Clin Rehabil. (2016) 30:432-40. doi: 10.1177/0269215515593611
53. Luo H, Cao C, Zhong J, Chen J, Cen Y. Adjunctive virtual reality for procedural pain management of burn patients during dressing change or physical therapy: a systematic review and meta-analysis of randomized controlled trials. Wound Repair Regenerat. (2019) 27:90-101. doi: 10.1111/wrr.1

54. Kampmann IL, Emmelkamp PM, Morina N. Meta-analysis of technologyassisted interventions for social anxiety disorder. J Anxiety Disord. (2016) 42:71-84. doi: 10.1016/j.janxdis.2016.06.007

55. Fodor LA, Cotet CD, Cuijpers P, Szamoskozi S, David D, Cristea IA. The effectiveness of virtual reality based interventions for symptoms of anxiety and depression: a meta-analysis. Sci Rep. (2018) 8:1-13. doi: 10.1038/s41598-018-28113-6

56. Bashiri A, Ghazisaeedi M, Shahmoradi L. The opportunities of virtual reality in the rehabilitation of children with attention deficit hyperactivity disorder: a literature review. Korean J Pediatr. (2017) 60:337-43. doi: $10.3345 / \mathrm{kjp} .2017 .60 .11 .337$

57. Chen Y-P, Lee S-Y, Howard AM. Effect of virtual reality on upper extremity function in children with cerebral palsy: a meta-analysis. Pediatric Phys Therapy. (2014) 26:289-300. doi: 10.1097/PEP.00000000000 00046

58. DiGennaro Reed FD, Hyman SR, Hirst JM. Applications of technology to teach social skills to children with autism. Res Autism Spectr Disord. (2011) 5:1003-10. doi: 10.1016/j.rasd.2011.01.022

59. Wainer AL, Ingersoll BR. The use of innovative computer technology for teaching social communication to individuals with autism spectrum disorders. Res Autism Spectr Disord. (2011) 5:96-107. doi: 10.1016/j.rasd.2010.08.002

60. Pennington RC. Computer-assisted instruction for teaching academic skills to students with autism spectrum disorders: a review of literature. Focus Autism Other Dev Disabil. (2010) 25:239-48. doi: $10.1177 / 1088357610378291$

61. Den WB, Sterkenburg PS. Self-controlled technologies to support skill attainment in persons with an autism spectrum disorder and/or an intellectual disability: a systematic literature review. Disabil Rehabil Assist Technol. (2015) 10:1-10. doi: 10.3109/17483107.2014.9 21248

62. Mesa-Gresa P, Gil-Gómez H, Lozano-Quilis J-A, Gil-Gómez J-A. Effectiveness of virtual reality for children and adolescents with autism spectrum disorder: an evidence-based systematic review. Sensors. (2018) 18:2486. doi: 10.3390/s18082486

63. Grynszpan O, Weiss PL, Perez-Diaz F, Gal E. Innovative technology-based interventions for autism spectrum disorders: a meta-analysis. Autism. (2014) 18:346-61. doi: 10.1177/1362361313476767

64. Bekele E, Wade J, Bian D, Fan J, Swanson A, Warren Z, et al. Multimodal adaptive social interaction in virtual environment (MASI-VR) for children with Autism spectrum disorders (ASD). In: 2016 IEEE Virtual Reality (VR). Greenville, SA: IEEE (2016). p. 121-30. doi: 10.1109/VR.2016.75 04695

65. Bozgeyikli L, Bozgeyikli E, Raij A, Alqasemi R, Katkoori S, Dubey R. Vocational rehabilitation of individuals with autism spectrum disorder with virtual reality. ACM Trans Accessible Comput. (2017) 10:3046786. doi: $10.1145 / 3046786$

66. Cox DJ, Brown T, Ross V, Moncrief M, Schmitt R, Gaffney G, et al. Can youth with autism spectrum disorder use virtual reality driving simulation training to evaluate and improve driving performance? an exploratory study. J Autism Dev Disord. (2017) 47:2544-55. doi: 10.1007/s10803-0173164-7

67. Dickinson K, Place M. The impact of a computer-based activity program on the social functioning of children with autistic spectrum disorder. Games Health J. (2016) 5:209-15. doi: 10.1089/g4h.2015. 0063

68. Escobedo L, Tentori M, Quintana E, Favela J, Garcia-Rosas D. Using augmented reality to help children with autism stay focused. IEEE Pervasive Comput. (2014) 13:38-46. doi: 10.1109/MPRV.2014.19

69. Lorenzo G, Pomares J, Lledo A. Inclusion of immersive virtual learning environments and visual control systems to support the learning of students with asperger syndrome. Comput Educ. (2013) 62:88-101. doi: 10.1016/j.compedu.2012.10.028 
70. Chen C-H, Lee I-J, Lin L-Y. Augmented reality-based self-facial modeling to promote the emotional expression and social skills of adolescents with autism spectrum disorders. Res Dev Disabil. (2015) 36:396-403. doi: 10.1016/j.ridd.2014.10.015

71. Cheng Y, Huang C-L, Yang C-S. Using a 3D immersive virtual environment system to enhance social understanding and social skills for children with autism spectrum disorders. Focus Autism Other Dev Disabil. (2015) 30:22236. doi: 10.1177/1088357615583473

72. Ke F, Im T. Virtual-reality-based social interaction training for children with high-functioning autism. J Educ Res. (2013) 106:441-61. doi: 10.1080/00220671.2013.832999

73. Wang M, Reid D. Using the virtual reality-cognitive rehabilitation approach to improve contextual processing in children with autism. Sci World J. (2013) 2013:716890. doi: 10.1155/2013/716890

74. Kim K, Rosenthal MZ, Gwaltney M, Jarrold W, Hatt N, McIntyre N, et al. A virtual joy-stick study of emotional responses and social motivation in children with autism spectrum disorder. J Autism Dev Disord. (2015) 45:3891-9. doi: 10.1007/s10803-014-2036-7

75. Parsons S. Learning to work together: designing a multi-user virtual reality game for social collaboration and perspective-taking for children with autism. Int J Child-Computer Interact. (2015) 6:28-38. doi: 10.1016/j.ijcci.2015.12.002

76. Morris SB, DeShon RP. Combining effect size estimates in meta-analysis with repeated measures and independent-groups designs. Psychol Methods. (2002) 7:105. doi: 10.1037/1082-989X.7.1.105

77. Morris SB. Estimating effect sizes from pretest-posttest-control group designs. Org Res Methods. (2008) 11:364-86. doi: 10.1177/1094428106291059

78. Moeyaert M, Ugille M, Beretvas SN, Ferron J, Bunuan R, Noortgate WV den. Methods for dealing with multiple outcomes in meta-analysis: a comparison between averaging effect sizes, robust variance estimation and multilevel meta-analysis. Int J Soc Res Methodol. (2017) 20:559-72. doi: 10.1080/13645579.2016.1252189

79. Cohen J. Statistical Power Analysis for the Social Sciences. Cambridge, MA: Academic press (1988).

80. Borenstein M, Higgins JPT, Hedges LV, Rothstein HR. Basics of metaanalysis: $I^{2}$ is not an absolute measure of heterogeneity. Res Synthesis Methods. (2017) 8:5-18. doi: 10.1002/jrsm.1230

81. Egger M, Smith GD, Schneider M, Minder C. Bias in meta-analysis detected by a simple, graphical test. BMJ. (1997) 315:629-34. doi: 10.1136/bmj.315.7109.629

82. Cheung MW-L. metaSEM: an $\mathrm{R}$ package for meta-analysis using structural equation modeling. Front Psychol. (2015) 5:1521. doi: 10.3389/fpsyg.2014.01521

83. Maskey M, Rodgers J, Ingham B, Freeston M, Evans G, Labus M, et al. Using virtual reality environments to augment cognitive behavioral therapy for fears and phobias in autistic adults. Autism Adulthood Challenges Manag. (2019) 1:134-45. doi: 10.1089/aut.2018.0019

84. Yuan SNV, Ip HHS. Using virtual reality to train emotional and social skills in children with autism spectrum disorder. London J Primary Care. (2018) 10:110-2. doi: 10.1080/17571472.2018.1483000

85. Chen C-H, Lee I-J, Lin L-Y. Augmented reality-based video-modeling storybook of non-verbal facial cues for children with autism spectrum disorder to improve their perceptions and judgments of facial expressions and emotions. Comput Hum Behav. (2016) 55:477-85. doi: 10.1016/j.chb.2015.09.033

86. Zhang L, Warren Z, Swanson A, Weitlauf A, Sarkar N. Understanding performance and verbal-communication of children with ASD in a collaborative virtual environment. J Autism Dev Disord. (2018) 48:2779-89. doi: 10.1007/s10803-018-3544-7

87. Kandalaft MR, Didehbani N, Krawczyk DC, Allen TT, Chapman SB. Virtual reality social cognition training for young adults with high-functioning autism. J Autism Dev Disord. (2013) 43:34-44. doi: $10.1007 /$ s10803-012-1544-6

88. Stichter JP, Laffey J, Galyen K, Herzog M. iSocial: delivering the social competence intervention for adolescents (SCI-A) in a 3D virtual learning environment for youth with high functioning autism. J Autism Dev Disord. (2014) 44:417-30. doi: 10.1007/s10803-013-1881-0
89. Maskey M, Lowry J, Rodgers J, McConachie H, Parr JR. Reducing specific phobia/fear in young people with autism spectrum disorders (ASDs) through a virtual reality environment intervention. PLoS ONE. (2014) 9:e100374. doi: 10.1371/journal.pone.0100374

90. Smith MJ, Fleming MF, Wright MA, Losh M, Humm LB, Olsen D, et al. Brief report: vocational outcomes for young adults with autism spectrum disorders at 6 months after virtual reality job interview training. J Autism Dev Disord. (2015) 45:3364-9. doi: 10.1007/s10803-015-2470-1

91. Smith MJ, Ginger EJ, Wright K, Wright MA, Taylor JL, Humm LB, et al. Virtual reality job interview training in adults with autism spectrum disorder. J Autism Dev Disord. (2014) 44:2450-63. doi: 10.1007/s10803-014-2113-y

92. Chen F, Wang L, Peng G, Yan N, Pan X. Development and evaluation of a 3$\mathrm{D}$ virtual pronunciation tutor for children with autism spectrum disorders. PLoS ONE. (2019) 14:e0210858. doi: 10.1371/journal.pone.0210858

93. Simoes M, Bernardes M, Barros F, Castelo-Branco M. Virtual travel training for autism spectrum disorder: proof-of-concept interventional study. JMIR Serious Games. (2018) 6:8428. doi: 10.2196/games.8428

94. Yang YJD, Allen T, Abdullahi SM, Pelphrey KA, Volkmar FR, Chapman SB. Brain responses to biological motion predict treatment outcome in young adults with autism receiving Virtual Reality Soc Cogn Train. (2017) 93:55-66. doi: 10.1016/j.brat.2017.03.014

95. Ke F, Moon J. Virtual collaborative gaming as social skills training for high-functioning autistic children. Br J Educ Technol. (2018) 49:728-41. doi: 10.1111/bjet.12626

96. Milne M, Luerssen MH, Lewis TW, Leibbrandt RE, Powers DMW. Development of a virtual agent based social tutor for children with autism spectrum disorders. In: The 2010 International Joint Conference on Neural Networks (IJCNN). Barcelona (2010). p. 1-9. doi: 10.1109/IJCNN.2010.5596584

97. Strickland DC, Coles CD, Southern LB. JobTIPS: a transition to employment program for individuals with autism spectrum disorders. J Autism Dev Disord. (2013) 43:2472-83. doi: 10.1007/s10803-013-1800-4

98. Bai Z, Blackwell AF, Coulouris G. Using augmented reality to elicit pretend play for children with autism. IEEE Trans Visual Comput Graph. (2015) 21:598-610. doi: 10.1109/TVCG.2014.2385092

99. Ip HHS, Wong SWL, Chan DFY, Byrne J, Li C, Yuan VSN, et al. Virtual reality enabled training for social adaptation in inclusive education settings for school-aged children with autism spectrum disorder (ASD). In: Cheung SKS, Kwok LF, Shang J, Wang A, Kwan R, editors. Blended Learning: Aligning Theory With Practices, ICBL 2016. City Univ Hong Kong; Caritas Inst Higher Educ; Hong Kong Soc Multimedia \& Image Comp; Hong Kong Pei Hua Educ Fdn. Beijing: Lecture Notes in Computer Science (2016). vol. 9757, p. 94-102. doi: 10.1007/978-3-319-41165-1_9

100. Bernardini S, Porayska-Pomsta K, Sampath H. Designing an intelligent virtual agent for social communication in autism. In: Proceedings of the Ninth AAAI Conference on Artificial Intelligence and Interactive Digital Entertainment. Boston, MA: AAAI Press (2014). p. 9-15. Available online at: http://dl.acm.org/citation.cfm?id=3014712.3014715 (accessed October 27, 2018).

101. Miller IT, Wiederhold BK, Miller CS, Wiederhold MD. Virtual reality air travel training with children on the autism spectrum: a preliminary report. Cyberpsychol Behav Soc Netw. 23:10-5. doi: 10.1089/cyber.2019.0093

102. Hedges LV, Tipton E, Johnson MC. Robust variance estimation in metaregression with dependent effect size estimates. Research Synthesis Methods. (2010) 1:39-65. doi: 10.1002/jrsm.5

103. Hong ER, Ganz JB, Mason R, Morin K, Davis JL, Ninci J, et al. The effects of video modeling in teaching functional living skills to persons with ASD: a meta-analysis of single-case studies. Res Dev Disabil. (2016) 57:158-69. doi: 10.1016/j.ridd.2016.07.001

104. Hong ER, Gong L, Ninci J, Morin K, Davis JL, Kawaminami S, et al. A meta-analysis of single-case research on the use of tablet-mediated interventions for persons with ASD. Res Dev Disabil. (2017) 70:198-214. doi: 10.1016/j.ridd.2017.09.013

105. Wilkinson KM, Hennig S. The state of research and practice in augmentative and alternative communication for children with developmental/intellectual disabilities. Mental Retard Dev Disabil Res Rev. (2007) 13:58-69. doi: $10.1002 / \operatorname{mrdd} .20133$ 
106. Cuijpers P, Weitz E, Cristea IA, Twisk J. Pre-post effect sizes should be avoided in meta-analyses. Epidemiol Psychiatric Sci. (2017) 26:364-8. doi: $10.1017 /$ S2045796016000809

Conflict of Interest: The authors declare that the research was conducted in the absence of any commercial or financial relationships that could be construed as a potential conflict of interest.
Copyright (c) 2021 Karami, Koushki, Arabgol, Rahmani and Vahabie. This is an open-access article distributed under the terms of the Creative Commons Attribution License (CC BY). The use, distribution or reproduction in other forums is permitted, provided the original author(s) and the copyright owner(s) are credited and that the original publication in this journal is cited, in accordance with accepted academic practice. No use, distribution or reproduction is permitted which does not comply with these terms. 